\title{
A mass-conserving lattice Boltzmann method with dynamic grid refinement for immiscible two-phase flows
}

\author{
Abbas Fakhari $^{\mathrm{a}}$, Martin Geier ${ }^{\mathrm{b}}$, Taehun Lee ${ }^{\mathrm{c}}$ \\ ${ }^{a}$ Department of Civil and Environmental Engineering and Earth Sciences, University of \\ Notre Dame, Notre Dame, IN 46556, USA \\ ${ }^{b} T U$ Braunschweig, Institute for Computational Modeling in Civil Engineering (iRMB), \\ TU-Braunschweig, Pockelsstr. 3, 38106 Braunschweig, Germany \\ ${ }^{c}$ Department of Mechanical Engineering, The City College of the City University of New \\ York, New York, NY 10031, USA
}

\begin{abstract}
A mass-conserving lattice Boltzmann method (LBM) for multiphase flows is presented in this paper. The proposed LBM improves the previous model [T. Lee and L. Liu (2010) Journal of Computational Physics 229, 8045-8063] in terms of mass conservation, speed-up, and efficiency, and also extends its capabilities for implementation on non-uniform grids. The presented model consists of a phase-field lattice Boltzmann equation (LBE) for tracking the interface between different fluids and a pressure-evolution LBM for recovering the hydrodynamic properties. In addition to the mass conservation property and the simplicity of the algorithm, the advantages of the current phase-field LBE are that it is an order of magnitude faster than the previous interface tracking LBE proposed by Lee and Liu [T. Lee and L. Liu (2010) Journal of Computational Physics 229, 8045-8063] and it needs less memory resources
\end{abstract}

Email address: afakhari@nd.edu (Abbas Fakhari) 
for data storage. Meanwhile, the pressure-evolution LBM is equipped with a multi-relaxation-time (MRT) collision operator to facilitate attainability of small relaxation rates thereby allowing simulation of multiphase flows at higher Reynolds numbers.

Additionally, we reformulate the presented MRT-LBM on nonuniform grids in the adaptive mesh refinement (AMR) framework. Various benchmark studies such as rising bubble and falling drop under buoyancy force, droplet splashing on a wet surface, and droplet coalescence onto a fluid interface are conducted to examine the accuracy and versatility of the proposed AMR-LBM. The proposed model is further validated by comparing the results with other LB models on uniform grids. A factor of about 20 savings in computational resources is achieved by using the proposed AMR-LBM. As a more demanding application, the Kelvin-Helmholtz instability (KHI) of a shear-layer flow is investigated for both density-matched and densitystratified binary fluids. The KHI results of the density-matched fluids are shown to be in good agreement with the benchmark AMR results based on the sharp-interface approach. When a density contrast between the two fluids exists, a typical chaotic structure in the flow field is observed at a Reynolds number of 10000, which indicates that the proposed model is a promising tool for direct numerical simulation of two-phase flows.

Keywords: mass-conserving lattice Boltzmann method, conservative phase-field, multiphase flow, adaptive mesh refinement, rising bubble, falling droplet, Kelvin-Helmholtz instability, dynamic grid adaptation 


\section{Introduction}

Sharp-interface models and diffuse-interface models [1, 2] are commonly used for numerical simulation of multiphase flows. The basic idea behind diffuse-interface models is to spread the interfacial region onto a finite number of grid points, which is typically around $3 \sim 6$ cells, and to employ a continuum (volumetric) force, instead of a singular Dirac $\delta$ function, to represent the surface tension. Numerically speaking, diffuse-interface methods are preferred over sharp-interface models, particularly in phase change problems [3], mainly because the computations for complex flows are performed much easier and irregular topological changes are readily captured by solving a phase-field equation. Diffuse-interface models are especially suitable for problems that involve interface disintegration and coalescence and in simulation of contact line dynamics.

Rooted in the kinetic theory, the lattice Boltzmann method (LBM) is a mesoscopic approach for simulation of fluid flows [4-6], and it is favorable because phase interface boundaries are essentially mesoscopic in nature. As a diffuse-interface model, the multiphase LBM has become a well established computational tool for numerical study of nonideal gases and complex fluids [7-11]. Several lattice Boltzmann (LB) models have been implemented and developed by other researchers. Among the existing two-phase LB models [12-21], the pressure-evolution LBM equipped with stencil discretization schemes has proved to be a promising method for studying the fluid dynamics of a variety of phenomena [21,22]. This model is an improved version of the model proposed by He and colleagues $[8,16]$ in that instead of using the Carnahan-Starling equation of state the chemical potential is used. In addi- 
tion, thanks to stable discretization stencils [18], the pressure-evolution LBM can handle large density differences and suppress spurious velocity currents to the machine precision [22].

The original pressure-evolution LBM, however, has two major drawbacks. First, as pointed out by Guo et al. [23], it suffers from the lack of mass conservation property. Second, it is restricted to moderate Reynolds numbers. The lack of mass conservation stems from the force imbalance in the interface tracking equation, which is due to the use of biased finite-difference (FD) scheme in the calculation of the gradient of the composition in the lattice Boltzmann equation (LBE) from which the Cahn-Hilliard equation [23] is derived. On the other hand, using a single-relaxation-time (SRT) collision operator [24] in the pressure-evolution equation for recovering the hydrodynamic properties limits the applicability of the model to relatively small Reynolds numbers.

From the computational point of view, simulation of multiphase flows on small scales is a challenging task. Given that the characteristic interfacial thickness is small and must be resolved by a sufficient number of computational points, higher resolution is required in the vicinity of the interface between different fluids. Solving the governing equations, which are usually in the form of partial differential equations, requires a high-fidelity numerical method. This is complicated by the fact that often we are dealing with highReynolds-number flows where higher grid resolution is desired, for example, in the vicinity of the interface between different fluids. Using a structured uniform grid throughout the computation domain could be costly and inefficient. Therefore, adaptive mesh refinement (AMR) techniques are employed. 
There are many AMR algorithms proposed in the literature. Berger and co-workers [25, 26] utilized rectangular, overlapping grid patches to solve the two-dimensional (2D) hyperbolic equations in the conservative form. De Zeeuw and Powel [27] proposed a 2D AMR scheme with a quad-tree data structure. To overcome the computational overhead of the tree-based approach, a fully threaded tree AMR was developed by Khokhlov [28]. Ji et al. [29] replaced the tree data structure with a hash table and proposed a cell-based AMR. Recently, another AMR algorithm without the need to maintain or modify a tree-type data structure was proposed by Fakhari and Lee [30].

There are also numerous AMR packages available [31-36]. Among them, the PARAmesh toolkit [31] is designed to extend an existing serial code for uniform grids to a parallel routine with dynamic grid adaptation. In the author's opinion, however, the PARAMESH is not written efficiently. Constructing a parallel AMR algorithm is yet another demanding topic. There is ongoing research in developing AMR algorithms in parallel and optimizing the load balancing techniques associated with them. Interested readers are referred to the excellent discourse by Rantakokko and Thun [37, chap. 5].

The LBM has also been incorporated into the AMR strategy. Tölke et al. [38] proposed an AMR-LBM for simulation of multiphase flows. They invoked a data structure consisted of the hierarchy of tree-type grids. $\mathrm{Yu}$ and Fan [39] used the PARAmesh toolkit [31] to implement an AMR-LBM for simulation of bubble rising due to buoyancy force. Inspired by the idea proposed in PARAmesh, Fakhari and Lee [40] developed a block-structured AMR algorithm for both standard LBM and FD-LBM using the cell-centered 
data structure.

Most of the existing LB models for interface tracking [41-43] are based on the Cahn-Hilliard $(\mathrm{CH})$ theory $[44,45]$. As it has been shown recently [46], using the $\mathrm{CH}$ equation causes small droplets to disappear once their radius is below a critical value. From the numerical point of view, another undesired feature of the $\mathrm{CH}$ models is the calculation of the Laplacian of the chemical potential. This implies the computation of a 4th-order derivative because the Laplacian of the composition or index function is required to compute the chemical potential. Consequently, another class of interface tracking equation, herein called the phase-field equation, is proposed by Sun and Beckermann [47]. The phase-field equation was recently reformulated in the conservative form by Chiu and Lin [48]. It requires only 2nd-order derivatives, which can be reduced to the calculation of 1st-order derivatives, or even no derivatives if central moments are invoked, in the LBM framework [49].

Recently, we developed a conservative phase-field LBE [49] which was more efficient than the original interface-tracking equation proposed in Ref. [21]. In the present study, we will combine the phase-field LBE for interface tracking equation [49] and the pressure-evolution LBM with a multiple-relaxationtime (MRT) collision operator $[50,51]$ to propose a mass-conserving LB model for simulation of two-phase flows at higher Reynolds numbers. In addition, we will extend the recently proposed vertex-centered AMR routine for single-phase flows [22] to a cell-centered AMR package for two-phase flows. Furthermore, we will propose an FD-based LBM, which is secondorder accurate in both time and space, for simulation of immiscible fluids 
on nonuniform grids. After extensive validation and assessment of the proposed AMR-LBM in terms of accuracy and efficiency, we will investigate the Kelvin-Helmholtz instability (KHI) of stratified fluids in a shear-layer flow at relatively high Reynolds numbers.

We will introduce the conservative phase-field LBE and recast the advection step on nonuniform grids in Sec. 2. The pressure-evolution MRTLBM for recovering the Navier-Stokes equations will be formulated in Sec. 3. Then, in Sec. 4, we will describe the AMR algorithm for two-phase flows and propose biquadratic interpolation schemes for cell-centered data points on block-structured adaptive grids followed by numerical validation in Sec. 5 . Application of the proposed AMR-LBM in the shear-layer instability of a vortex sheet will be presented in Sec. 6. Finally, we will summarize the paper in Sec. 7 .

\section{Conservative phase-field LBE}

The conservative phase-field equation for tracking the interface in the incompressible two-phase flow reads as [48]

$$
\frac{\partial \phi}{\partial t}+\nabla \cdot \phi \mathbf{u}=\nabla \cdot\left[M\left(\nabla \phi-\frac{1-4\left(\phi-\phi_{a v g}\right)^{2}}{W} \mathbf{n}\right)\right]
$$

where $\phi$ is the phase-field variable that indicates each phase such that $\phi_{\text {avg }}=$ $\left(\phi_{l}+\phi_{h}\right) / 2=0$ gives us the interface location $\left(\phi_{l}=0\right.$ and $\phi_{h}=1$ are used in this study), $t$ stands for the time, $\mathbf{u}$ is the macroscopic velocity vector, $M$ is the mobility, $W$ is the interface width, and $\mathbf{n}$ is the vector normal to the interface

$$
\mathbf{n}=\frac{\nabla \phi}{|\nabla \phi|}
$$


At equilibrium, the phase-field variable along the direction $\xi$ normal to an interface located at $\xi=0$ assumes a hyperbolic tangent profile

$$
\phi(\xi)=\phi_{\text {avg }}+\frac{\phi_{h}-\phi_{l}}{2} \tanh \left(\frac{2 \xi}{W}\right)
$$

Equation (1) will be solved using the following LBE, which is inherently conservative [49]:

$$
\frac{\partial h_{\alpha}}{\partial t}+\mathbf{e}_{\alpha} \cdot \nabla h_{\alpha}=-\frac{h_{\alpha}-h_{\alpha}^{e q}}{\lambda_{\phi}}
$$

where $h_{\alpha}$ and $\lambda_{\phi}$ are, respectively, the distribution function and the relaxation parameter that are related to the phase-field variable, and $\mathbf{e}_{\alpha}$ is the microscopic velocity set. For the 2D nine-velocity lattice (D2Q9) used in the present study $\mathbf{e}_{0}=(0,0) ; \mathbf{e}_{\alpha}=c\left(\cos \theta_{\alpha}, \sin \theta_{\alpha}\right)$ for $\alpha=1-4$; and $\mathbf{e}_{\alpha}=\sqrt{2} c\left(\cos \theta_{\alpha}, \sin \theta_{\alpha}\right)$ for $\alpha=5-8$, where $c=\delta x / \delta t=1$ is the lattice speed with $\delta x$ being the lattice length scale [4] and $\delta t$ being the lattice time scale. The lattice time scale is identical to the time step in our simulations.

The phase-field distribution function at equilibrium is [49]

$$
h_{\alpha}^{e q}=\phi \Gamma_{\alpha}+\theta w_{\alpha} \mathbf{e}_{\alpha} \cdot \mathbf{n}
$$

in which

$$
\Gamma_{\alpha}=w_{\alpha}\left[1+\frac{\mathbf{e}_{\alpha} \cdot \mathbf{u}}{c_{s}^{2}}+\frac{\left(\mathbf{e}_{\alpha} \cdot \mathbf{u}\right)^{2}}{2 c_{s}^{4}}-\frac{\mathbf{u} \cdot \mathbf{u}}{2 c_{s}^{2}}\right]
$$

where $c_{s}=c / \sqrt{3}$ is the lattice speed of sound and $w_{\alpha}$ is the weight coefficient set with $w_{0}=4 / 9 ; w_{1-4}=1 / 9 ;$ and $w_{5-8}=1 / 36$ for the D2Q9 lattice [4]. The last term in Eq. (5) accounts for anti-diffusion where

$$
\theta=\frac{M}{c_{s}^{2}}\left[\frac{1-4\left(\phi-\phi_{a v g}\right)^{2}}{W}\right]
$$


As it has been shown before [30], we can split Eq.(4) into two steps;

1- Collision:

$$
\hat{h}_{\alpha}=h_{\alpha}-\frac{h_{\alpha}-h_{\alpha}^{e q}}{\tau_{\phi}^{\prime}+0.5}
$$

where $\tau_{\phi}^{\prime}=\lambda_{\phi} / \delta t$ is the dimensionless relaxation time for the phase-field variable, and

2- Advection:

$$
\frac{\partial \hat{h}_{\alpha}}{\partial t}+\mathbf{e}_{\alpha} \cdot \nabla \hat{h}_{\alpha}=0
$$

The mobility is related to the relaxation time by

$$
M=\tau_{\phi}^{\prime} c_{s}^{2} \delta t
$$

In order to retain the second-order accuracy of the LBM in time and space, we employ the following Lax-Wendroff FD scheme to solve the advection equation on nonuniform grids:

$$
\begin{aligned}
h_{\alpha}(\mathbf{x}, t+\delta t) & =\hat{h}_{\alpha}(\mathbf{x}, t)-C\left[\hat{h}_{\alpha}(\mathbf{x}, t)-\hat{h}_{\alpha}\left(\mathbf{x}-\mathbf{e}_{\alpha} \Delta x / c, t\right)\right] \\
& -\frac{1}{2} C(1-C)\left[\hat{h}_{\alpha}\left(\mathbf{x}+\mathbf{e}_{\alpha} \Delta x / c, t\right)-2 \hat{h}_{\alpha}(\mathbf{x}, t)+\hat{h}_{\alpha}\left(\mathbf{x}-\mathbf{e}_{\alpha} \Delta x / c, t\right)\right]
\end{aligned}
$$

where $\mathbf{x}$ is the position vector, $\Delta x$ is the grid spacing, and $C=c \delta t / \Delta x$ is the Courant-Friedrichs-Lewy (CFL) number. As can be seen in the above equation, when the CFL number is equal to one $(C=1)$ the Lax-Wendroff approximation of the advection step gives us the exact solution, namely the perfect shift

$$
h_{\alpha}(\mathbf{x}, t+\delta t)=\hat{h}_{\alpha}\left(\mathbf{x}-\mathbf{e}_{\alpha} \Delta x / c, t\right)
$$

This is the case when the spatial resolution is at its finest, i.e. when $\Delta x=$ $\delta t=1$, and it is beneficial because the perfect shift solution eliminates any 
numerical error due to dispersion or dissipation stemming from the LaxWendroff scheme.

After performing the advection step, the phase-field is calculated by taking the zeroth moment of the distribution function

$$
\phi=\sum_{\alpha=0}^{8} h_{\alpha}
$$

And then the density $\rho$ is updated by

$$
\rho=\rho_{l}+\frac{\phi-\phi_{l}}{\phi_{h}-\phi_{l}}\left(\rho_{h}-\rho_{l}\right)
$$

where $\rho_{l}$ and $\rho_{h}$ are the bulk densities of the light and heavy fluids, respectively.

The only thing left to determine is the calculation of the vector normal to the interface in Eq. (2). In this study we use the 2nd-order isotropic central difference to calculate the gradient of the phase-field

$$
\nabla \phi=\frac{3}{2 c \Delta x} \sum_{\alpha=1}^{8} \mathbf{e}_{\alpha} w_{\alpha}\left[\phi\left(\mathbf{x}+\mathbf{e}_{\alpha} \Delta x / c\right)-\phi\left(\mathbf{x}-\mathbf{e}_{\alpha} \Delta x / c\right)\right]
$$

Another approach for computation of the interface normal is to invoke central moments to approximate Eq. (2) according to [49]:

$$
\mathbf{n}=-\frac{\mathbf{m}_{c}}{\left|\mathbf{m}_{c}\right|}
$$

where

$$
\mathbf{m}_{c}=\frac{1}{c} \sum_{\alpha=0}^{8} h_{\alpha}\left(\mathbf{e}_{\alpha}-\mathbf{u}\right)
$$

The benefit of using the central moments to calculate the interface normal is that the collision step becomes completely local with no derivatives involved. On the other hand, the FD approximation of the interface normal gives us 
slightly more accurate and more stable solution mainly because the momentbased approach is very sensitive to grid scale oscillations [49]. Since the derivatives of the phase-field variable will appear in the pressure-evolution equation (see Sec.3), we choose isotropic FD [18] for the computation of the interface normal. Ref. [49] can be consulted for further details and derivation of the moment-based phase-field LBE. Therein, the comparison between the moment-based and the FD-based approaches is also provided.

\section{Pressure-evolution MRT-LBM}

The discrete Boltzmann equation for nonideal fluids in its general form can be written as [8]:

$$
\frac{\partial f_{\alpha}}{\partial t}+\mathbf{e}_{\alpha} \cdot \nabla f_{\alpha}=-\boldsymbol{\Lambda}\left(f_{\alpha}-f_{\alpha}^{e q}\right)+\frac{\left(\mathbf{e}_{\alpha}-\mathbf{u}\right) \cdot \mathbf{F}}{c_{s}^{2}} \Gamma_{\alpha}
$$

where $f_{\alpha}$ is the particle distribution function, $\boldsymbol{\Lambda}$ is the generalized collision operator, $\mathbf{F}$ is the forcing term, and the equilibrium distribution function is

$$
f_{\alpha}^{e q}=\rho \Gamma_{\alpha}
$$

The force can be written as

$$
\mathbf{F}=-\nabla\left(p-\rho c_{s}^{2}\right)+\mathbf{F}_{s}+\mathbf{F}_{b}
$$

in which $p$ is the pressure, $\mathbf{F}_{b}$ is the body force, and the surface tension force in its original form is given by [21]

$$
\mathbf{F}_{s}=-\phi \nabla \mu_{\phi}
$$

where the chemical potential is

$$
\mu_{\phi}=4 \beta\left(\phi-\phi_{l}\right)\left(\phi-\phi_{h}\right)\left(\phi-\phi_{a v g}\right)-\kappa \nabla^{2} \phi
$$


in which $\beta$ and $\kappa$ are related to the surface tension $\sigma$ and interface width $W$ by $\beta=12 \sigma / W$ and $\kappa=3 \sigma W / 2$. In this study, we use the following continuum surface tension form instead [52]

$$
\mathbf{F}_{s}=\mu_{\phi} \nabla \phi
$$

This has two imminent advantages: first, computing the derivative of the chemical potential in Eq. (21) is avoided, which enhances the locality of the collision step; second, in addition to reducing the memory overhead due to storing a vector variable for the gradient of the chemical potential, the numerical stiffness in the calculation of $\nabla \mu_{\phi}$, which is proportional to $\nabla \nabla^{2}$, is diminished. It is worth mentioning that we can also use another form of the continuum surface tension force with no chemical potential involved, $\mathbf{F}_{s}=-\kappa|\nabla \phi|^{2} \mathbf{n} \nabla \cdot \mathbf{n}$, as proposed by Kim [52]

Introducing a new distribution function $g_{\alpha}=f_{\alpha} c_{s}^{2}+w_{\alpha}\left(p-\rho c_{s}^{2}\right)$ and substituting it into Eq. (18), while having in mind that in the incompressible limit the Mach number of the flow is very small $(\mathrm{Ma} \ll 1)$ and therefore $\mathbf{u} \cdot \nabla p \sim \mathcal{O}\left(\mathrm{Ma}^{3}\right)$ is negligible, leads to

$\frac{\partial g_{\alpha}}{\partial t}+\mathbf{e}_{\alpha} \cdot \nabla g_{\alpha}=-\boldsymbol{\Lambda}\left(g_{\alpha}-g_{\alpha}^{e q}\right)+\left(\mathbf{e}_{\alpha}-\mathbf{u}\right) \cdot\left[\left(\Gamma_{\alpha}-w_{\alpha}\right) \nabla \rho c_{s}^{2}+\Gamma_{\alpha}\left(\mu_{\phi} \nabla \phi+\mathbf{F}_{b}\right)\right]$

where $g_{\alpha}^{e q}=f_{\alpha}^{e q} c_{s}^{2}+w_{\alpha}\left(p-\rho c_{s}^{2}\right)$. Noting that $\phi_{l}=0$ and $\phi_{h}=1$, according to Eq. (14)

$$
\nabla \rho=\frac{\partial \rho}{\partial \phi} \nabla \phi=\left(\rho_{h}-\rho_{l}\right) \nabla \phi
$$


and Eq. (24) can be further simplified to

$$
\begin{aligned}
\frac{\partial g_{\alpha}}{\partial t}+\mathbf{e}_{\alpha} \cdot \nabla g_{\alpha}=-\boldsymbol{\Lambda}\left(g_{\alpha}-g_{\alpha}^{e q}\right) & +\left[\left(\Gamma_{\alpha}-w_{\alpha}\right)\left(\rho_{h}-\rho_{l}\right) c_{s}^{2}+\Gamma_{\alpha} \mu_{\phi}\right]\left(\mathbf{e}_{\alpha}-\mathbf{u}\right) \cdot \nabla \phi \\
+ & \Gamma_{\alpha}\left(\mathbf{e}_{\alpha}-\mathbf{u}\right) \cdot \mathbf{F}_{b}
\end{aligned}
$$

Similar to the approach presented in Sec. 2, we can split the above equation into collision and advection. The final form of the collision step reads as

$$
\begin{aligned}
\hat{g}_{\alpha}(\mathbf{x}, t) & =\bar{g}_{\alpha}(\mathbf{x}, t)-\left.\mathbf{M}^{-1} \hat{\mathbf{S M}}\left(\bar{g}_{\alpha}-\bar{g}_{\alpha}^{e q}\right)\right|_{(\mathbf{x}, t)} \\
& +\left.\delta t\left[\left(\Gamma_{\alpha}-w_{\alpha}\right)\left(\rho_{h}-\rho_{l}\right) c_{s}^{2}+\Gamma_{\alpha} \mu_{\phi}\right]\left(\mathbf{e}_{\alpha}-\mathbf{u}\right) \cdot \nabla^{M} \phi\right|_{(\mathbf{x}, t)} \\
& +\left.\delta t \Gamma_{\alpha}\left(\mathbf{e}_{\alpha}-\mathbf{u}\right) \cdot \mathbf{F}_{b}\right|_{(\mathbf{x}, t)}
\end{aligned}
$$

where $\mathbf{M}$ and $\mathbf{M}^{-1}$ are the orthogonal transformation matrix and its inverse, respectively [50], $\hat{\mathbf{S}}=\boldsymbol{\Lambda} \delta t$ is the diagonal relaxation matrix, and the following transformation was used to maintain an explicit scheme

$$
\begin{aligned}
\bar{g}_{\alpha}=g_{\alpha} & +\frac{1}{2} \mathbf{S}\left(g_{\alpha}-g_{\alpha}^{e q}\right) \\
& -\frac{\delta t}{2}\left[\left(\Gamma_{\alpha}-w_{\alpha}\right)\left(\rho_{h}-\rho_{l}\right) c_{s}^{2}+\Gamma_{\alpha} \mu_{\phi}\right]\left(\mathbf{e}_{\alpha}-\mathbf{u}\right) \cdot \nabla^{C} \phi \\
& -\frac{\delta t}{2} \Gamma_{\alpha}\left(\mathbf{e}_{\alpha}-\mathbf{u}\right) \cdot \mathbf{F}_{b}
\end{aligned}
$$

where the modified equilibrium distribution function reads as

$\bar{g}_{\alpha}^{e q}=g_{\alpha}^{e q}-\frac{\delta t}{2}\left[\left(\Gamma_{\alpha}-w_{\alpha}\right)\left(\rho_{h}-\rho_{l}\right) c_{s}^{2}+\Gamma_{\alpha} \mu_{\phi}\right]\left(\mathbf{e}_{\alpha}-\mathbf{u}\right) \cdot \nabla^{C} \phi-\frac{\delta t}{2} \Gamma_{\alpha}\left(\mathbf{e}_{\alpha}-\mathbf{u}\right) \cdot \mathbf{F}_{b}$

The superscripts $C$ and $M$ on the gradient operators stand for central and mixed finite differences, respectively, which are explicitly given by

$$
\begin{aligned}
\left.\frac{1}{c} \mathbf{e}_{\alpha} \cdot \nabla^{C} \phi\right|_{\mathbf{x}, t} & =\frac{\phi\left(\mathbf{x}+\mathbf{e}_{\alpha} \Delta x / c, t\right)-\phi\left(\mathbf{x}-\mathbf{e}_{\alpha} \Delta x / c, t\right)}{2 \Delta x} \\
\left.\frac{1}{c} \mathbf{e}_{\alpha} \cdot \nabla^{M} \phi\right|_{\mathbf{x}, t} & =\frac{-\phi\left(\mathbf{x}+2 \mathbf{e}_{\alpha} \Delta x / c, t\right)+5 \phi\left(\mathbf{x}+\mathbf{e}_{\alpha} \Delta x / c, t\right)-3 \phi(\mathbf{x}, t)-\phi\left(\mathbf{x}-\mathbf{e}_{\alpha} \Delta x / c, t\right)}{4 \Delta x}
\end{aligned}
$$


The above lattice Boltzmann equations recover the following Navier-Stokes equation for multiphase flows

$$
\rho\left(\frac{\partial \mathbf{u}}{\partial t}+\mathbf{u} \cdot \nabla \mathbf{u}\right)=-\nabla p+\nabla \cdot[\mu(\nabla \mathbf{u}+\mathbf{u} \nabla)]+\mathbf{F}_{s}+\mathbf{F}_{b}
$$

In this paper, the diagonal relaxation matrix is $\hat{\mathbf{S}}=\operatorname{diag}\left(1,1,1,1,1,1,1, s_{\nu}, s_{\nu}\right)$, where $s_{\nu}=1 /\left(\tau^{\prime}+0.5\right)$, and the kinematic viscosity is related to the relaxation time by

$$
\nu=\tau^{\prime} c_{s}^{2} \delta t
$$

As explained by Lee and Lin [21], the relaxation time should be calculated using an inverse interpolation from the phase field

$$
\frac{1}{\tau^{\prime}}=\frac{1}{\tau_{l}^{\prime}}+\frac{\phi-\phi_{l}}{\phi_{h}-\phi_{l}}\left(\frac{1}{\tau_{h}^{\prime}}-\frac{1}{\tau_{l}^{\prime}}\right)
$$

where $\tau_{l}^{\prime}$ and $\tau_{h}^{\prime}$ are the relaxation times for the light and heavy fluids, respectively. We adapted the primed notation to distinguish the relaxation time used in our study $\tau^{\prime}$ from the commonly used relaxation time in the literature $\tau=\tau^{\prime}+0.5$.

After the collision step, the advection is carried out using the Lax-Wendroff scheme

$$
\begin{aligned}
\bar{g}_{\alpha}(\mathbf{x}, t+\delta t) & =\hat{g}_{\alpha}(\mathbf{x}, t)-C\left[\hat{g}_{\alpha}(\mathbf{x}, t)-\hat{g}_{\alpha}\left(\mathbf{x}-\mathbf{e}_{\alpha} \Delta x / c, t\right)\right] \\
& -\frac{1}{2} C(1-C)\left[\hat{g}_{\alpha}\left(\mathbf{x}+\mathbf{e}_{\alpha} \Delta x / c, t\right)-2 \hat{g}_{\alpha}(\mathbf{x}, t)+\hat{g}_{\alpha}\left(\mathbf{x}-\mathbf{e}_{\alpha} \Delta x / c, t\right)\right]
\end{aligned}
$$

Then, the hydrodynamic properties are calculated by

$$
\begin{gathered}
\mathbf{u}=\frac{1}{\rho c_{s}^{2}}\left(\sum_{\alpha} \bar{g}_{\alpha} \mathbf{e}_{\alpha}+\frac{\delta t}{2}\left(\mu_{\phi} \nabla^{C} \phi+\mathbf{F}_{b}\right)\right) \\
p=\sum_{\alpha} \bar{g}_{\alpha}+\frac{\delta t}{2}\left(\rho_{h}-\rho_{l}\right) c_{s}^{2} \mathbf{u} \cdot \nabla^{C} \phi
\end{gathered}
$$




\section{Adaptive mesh refinement}

In this section, we extend the previously proposed AMR routine [30, 40] to a two-phase flow solver. The skeleton of our AMR routine is unchanged with subtle differences in filling buffer layers and using interpolation schemes. For the sake of self-consistency, however, the two-phase AMR algorithm will be described briefly in 2D.

The simulations begin by covering the computational domain with a single or, depending on the geometry, multiple base (or root) block(s). All blocks are structured and self-similar in the sense that (in 2D) they consist of $n_{x} \times n_{y}$ cells with different aspect ratios, or grid spacings, characterized by their refinement level $l$. The base block is the coarsest with $l=0$. The grid spacings of the blocks are related to their refinement level and the size of the root block $\left(L_{x} \times L_{y}\right)$ by

$$
\Delta x=\frac{L_{x}}{n_{x} \times 2^{l}}=\frac{L_{y}}{n_{y} \times 2^{l}}
$$

where in the present study $n_{x}=n_{y}=8$ is used.

The grid refinement procedure starts by refining the base block, and the subsequently generated blocks, creating the AMR hierarchy. Each time a block is refined, four child blocks (eight child blocks in 3D) are created and the grid resolution doubles. The refinement is triggered based on a so-called error estimator or refinement criterion (more on this follows later on in this section). Once the error within a certain block is above a threshold value $\epsilon_{r}$, it will be flagged for refinement. In contrary, if the error is below a prescribed value $\epsilon_{d}$ the block will be flagged for derefinement. The block refinement stops once a prescribed maximum refinement level $l_{\max }$ is reached. The 
computations are performed only on the leaf blocks which do not have any child blocks. It is worth noting that on the finest blocks where $l=l_{\max }$ the CFL number is equal to one $(\delta t=\Delta x=1)$. This mean that the solution to the advection equation Eq. (9) is perfect shift, as is the case in the standard LBM.

For each block, which is a derived type in ForTRAN, we use integer numbers to store its refinement level, its parent ID, and its geometrical indices. Unlike previous AMR algorithms [26, 28, 29], we keep track of the neighbors and child blocks of a given block by using an integer pointer to assign its children IDs as well as a nested pointer array to determine its neighboring blocks. Thereby the need to maintain, search, or modify a tree data structure is avoided [30].

\subsection{Filling buffer layers}

After establishing the connectivity information for inter-block and intrablock communications, each block should grant access to a portion of the data on the adjacent blocks. This is accomplished by using buffer layers around each block. As displayed in Fig. 1, we encounter three scenarios for filling the buffer cells of each block. The simplest case is when the two neighboring blocks are at the same level as shown in Fig. 1(a). The corresponding buffer

cells of block $A$ are easily filled by a direct copy from the available data cells in block $B$.

The second case is when the neighbor of block $A$ has child blocks, as depicted in Fig. 1(b). The following biquadratic formula, as illustrated in 


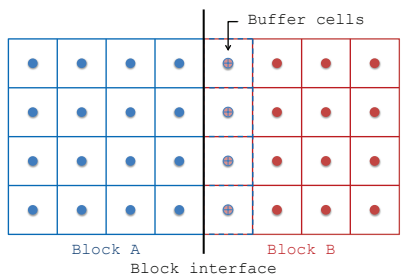

(a)

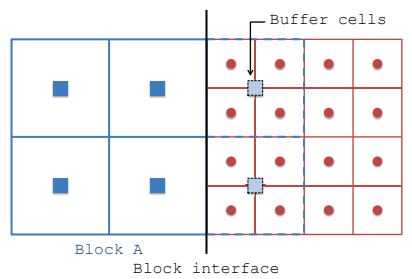

(b)

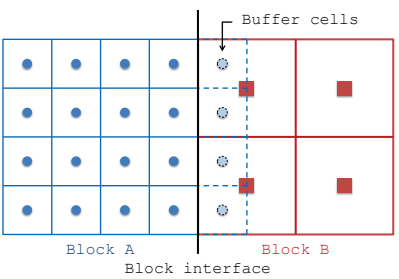

(c)

Figure 1: Different cases in filling the buffer cells of block $A$ to the left. (a) $l_{B}=l_{A}$ and block $\mathrm{B}$ is a leaf block; (b) $l_{B}=l_{A}$ but block $\mathrm{B}$ is refined (has children); (c) $l_{B}=l_{A}-1$.

Fig. 2(a), is used to fill the buffer cells of coarse blocks

$$
\begin{aligned}
f(I, J) & =[9 f(i, j)+18 f(i+1, j)-3 f(i+2, j) \\
& +18 f(i, j+1)+36 f(i+1, j+1)-6 f(i+2, j+1) \\
& -3 f(i, j+2)-6 f(i+1, j+2)+f(i+2, j+2)] / 64+\mathcal{O}\left(\Delta x^{3}\right)
\end{aligned}
$$

In the equation above, $f$ can be the phase field $\phi$, which is needed for the collision step, or the distribution functions $\hat{h}_{\alpha}$ and $\hat{g}_{\alpha}$, which are needed to perform the advection step. Of note is that, on all sides of each block, two layers of buffer cells are needed for $\phi$ while only one layer is required for $\hat{h}_{\alpha}$ and $\hat{g}_{\alpha}$.

The last case, as shown in Fig. 1(c), is when the resolution of block $A$ is one level finer than its neighboring block. For consistency in filling two layers of buffer cells in both orthogonal and diagonal directions, we suggest 


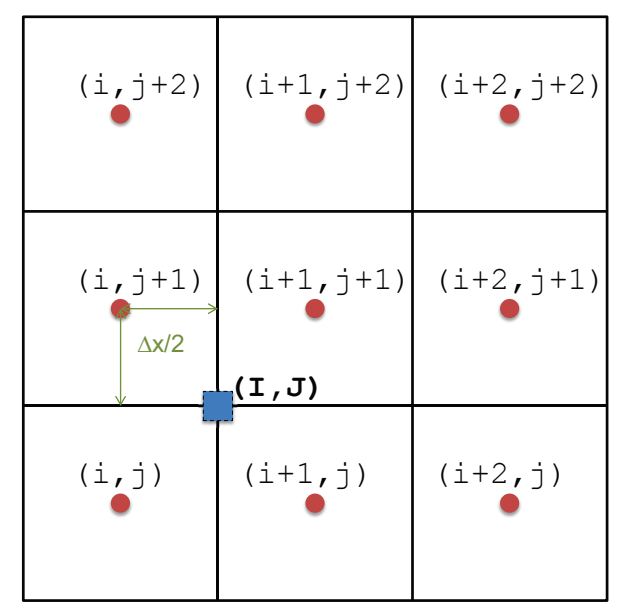

(a)

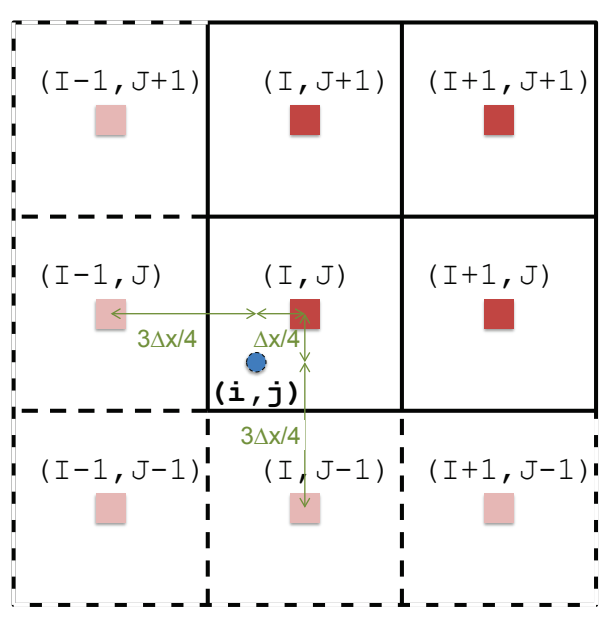

(b)

Figure 2: The data cells that are used for interpolation. (a) filling the buffer cell of a coarse block (blue square) from the available data points (red circles) on the fine block; (b) filling the buffer cell of a fine block (blue circle) from the data points (red squares) of the coarse block.

the following biquadratic interpolation, as illustrated in Fig. 2(b)

$$
\begin{aligned}
f(i, j) & =[25 f(I-1, J-1)+150 f(I, J-1)-15 f(I+1, J-1) \\
& +150 f(I-1, J)+900 f(I, J)-90 f(I+1, J) \\
& -15 f(I-1, J+1)-90 f(I, J+1)+9 f(I+1, J+1)] / 1024+\mathcal{O}\left(\Delta x^{3}\right)
\end{aligned}
$$

It is worth noting that in filling the buffer layers of fine blocks we might use the buffer cells of the adjacent coarser blocks, as denoted in Fig. 2(b) by dashed lines. Therefore, the buffer cells of the coarsest blocks are filled first followed by the blocks at the next refinement level and so forth.

Invoking a, at least, third-order accurate interpolation scheme has been proven essential in obtaining a spatially second-order accurate solution [53, 
$22,53]$, and that is the reason we use biquadratic interpolations. In the case that a block is next to an external boundary, instead of filling the buffer layers we apply the relevant boundary conditions by invoking ghost cells.

\subsection{Refinement criteria}

A good error estimator is essential for triggering refinement and derefinement in dynamic grid adaptation routines. Finding a unique error estimator that gives reasonable accuracy and efficiency for different types of flow fields is not straightforward. For single-phase flows, we found that vorticity-based criterion is sufficiently efficient and reliable [54]. For two-phase flows, we examined different refinement criteria based on the vorticity, interface curvature, and phase-field gradients or their combinations. We found that the

following error estimator $\epsilon$, among others, gives us reasonably efficient and accurate results for the simulation of multiphase flows in different configurations:

$$
\epsilon=|\nabla \phi|=\sqrt{\left(\frac{\partial \phi}{\partial x}\right)^{2}+\left(\frac{\partial \phi}{\partial y}\right)^{2}}
$$

In the following simulations we use the above mentioned error estimator with the following refinement criteria $\epsilon_{r}=0.002$ and $\epsilon_{d}=0.001$ unless otherwise specified.

\section{Numerical results}

In this section, several benchmark studies are considered to assess the proposed AMR-LBM. Two important parameters are often used in diffuseinterface models, the numerical Péclet number

$$
\mathrm{Pe}=\frac{U_{0} W}{M}
$$


where $U_{0}$ is the reference velocity, and the Cahn number

$$
\mathrm{Cn}=\frac{W}{L_{0}}
$$

where $L_{0}$ is the reference length scale. The Péclet number can be used to identify the relative strength between advection and diffusion while the Cahn number is a measure for convergence towards sharp-interface limit [55, 56].

\subsection{Rising bubble}

Evolution of a bubble rising due to buoyancy force has been studied by many authors [57-59]. We also consider the motion of a bubble in a rectangular channel. Initially, a circular bubble with diameter $D=L_{0} / 5$ is placed at $\left(L_{0} / 2, L_{0} / 2\right)$ in a rectangular domain of length $L_{0}$ and height $3 L_{0}$. The link bounce-back boundary condition is applied at the bottom and top, while periodic boundary conditions are used at the lateral boundaries. The volumetric buoyancy force $\mathbf{F}_{b}=-\left(\rho-\rho_{h}\right) G_{y} \hat{\mathbf{j}}$, where $G_{y}$ is the magnitude of the gravitational acceleration in the $y$-direction, is applied to the fluids. Dimensionless analysis suggests four important groups: the density ratio $\rho_{h} / \rho_{l}$, the viscosity ratio $\mu_{h} / \mu_{l}$, the gravity Reynolds number

$$
\operatorname{Re}_{h}=\frac{\sqrt{G_{y} \rho_{h}\left(\rho_{h}-\rho_{l}\right) D^{3}}}{\mu_{h}}
$$

and the Eötvös (Bond) number

$$
\mathrm{Eo}=\frac{G_{y}\left(\rho_{h}-\rho_{l}\right) D^{2}}{\sigma}
$$

In the literature usually the Morton number of the flow is used

$$
\mathrm{Mo}=\frac{G_{y}\left(\rho_{h}-\rho_{l}\right) \mu_{h}^{4}}{\sigma^{3} \rho_{h}^{2}}
$$


The dimensionless time is also defined by

$$
t^{*}=t \sqrt{\frac{G_{y}}{D}}
$$

The reference velocity scale need in the Péclet number can be chosen as $U_{0}=\sqrt{G_{y} D}$ for the gravity-driven flows.

Three sets of simulations are conducted at three different Eötvös numbers: $\mathrm{Eo}=5, \mathrm{Eo}=20$, and $\mathrm{Eo}=200$. The density and viscosity ratios are fixed at 1000 and 100, respectively, and the gravity Reynolds number is set to 100. The numerical parameters are $\mathrm{Pe}=1.1$ and $\mathrm{Cn}=0.010$, and the $\mathrm{AMR}$ simulation results are shown in Fig. 3. At the lowest Eötvös number a 2D ellipsoidal bubble is observed and at a medium Eötvös value a 2D sphericalcap bubble is seen while at a high enough Eötvös number a skirted bubble is formed. These patterns are consistent with the shape regime map provided by Clift et al. [57] for the 3D bubbles.

In order to assess the accuracy and reliability of the proposed AMRLBM, comparison between the AMR result and the uniform-grid solution is made and the results are shown in Fig. 4 at $t^{*}=12$. As it can be seen, the current AMR-LBM results are in satisfactory agreement with the uniformgrid solutions. Of note is that the bubbles in Fig. 4 have traveled slightly more distance when the AMR-LBM is used. The main reason for this discrepancy is that the Lax-Wendroff scheme damps the pressure waves that reflect back into the domain from the top wall. This can be further inferred from Fig. 5, which shows the average Reynolds number of the rising bubble $\operatorname{Re}_{r}=\rho_{h} u_{r} D / \mu_{h}$, where $u_{r}$ is the rise velocity, versus the dimensionless time. As it can be seen in Fig. 5, the average Reynolds number of the bubble using the AMR and uniform-grid solutions coincide until about $t^{*}=6$, whereafter 


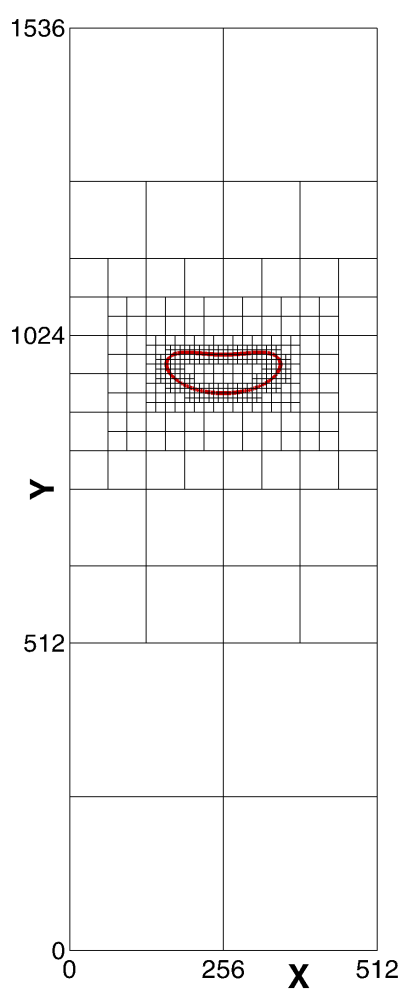

(a) $\mathrm{Eo}=5$

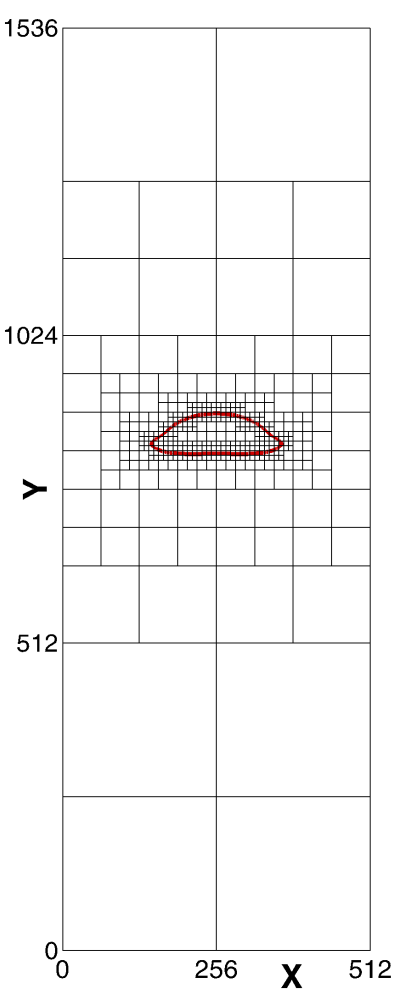

(b) $\mathrm{Eo}=20$

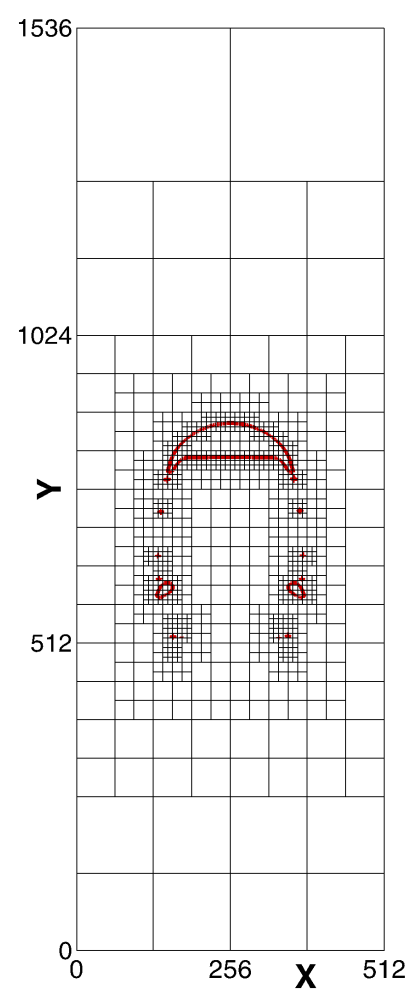

(c) $\mathrm{Eo}=200$

Figure 3: AMR-LBM simulations of rising bubble under buoyancy force. $\rho_{h} / \rho_{l}=1000$, $\mu_{h} / \mu_{l}=100, \operatorname{Re}_{h}=100$, and $t^{*}=12$. (a) Eo $=5\left(\mathrm{Mo}=1.2 \times 10^{-6}\right) ;(\mathrm{b})$ Eo $=20$ $\left(\mathrm{Mo}=8.0 \times 10^{-5}\right) ;(\mathrm{c})$ Eo $=200\left(\mathrm{Mo}=8.0 \times 10^{-2}\right)$.

the AMR-LBM predicts slightly higher rise velocity for the bubble. The relative error in the terminal velocity of the rising bubble was found to be less than $1 \%$ when comparing the AMR-LBM with the uniform-grid results.

We also carried out the AMR simulations of rising bubble at a higher grid resolution by increasing the maximum refinement level from $l_{\max }=6$ to $l_{\max }=7$ while keeping the Cahn number constant. The comparison between the AMR-LBM results at two different grid resolutions is shown in Fig. 6. 


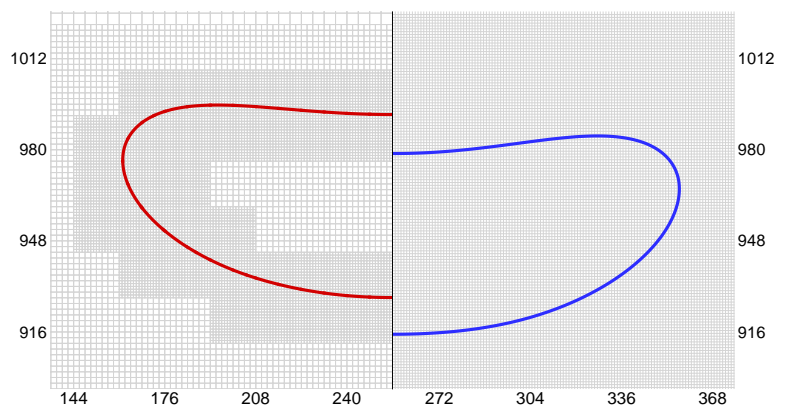

(a) $\mathrm{Eo}=5$

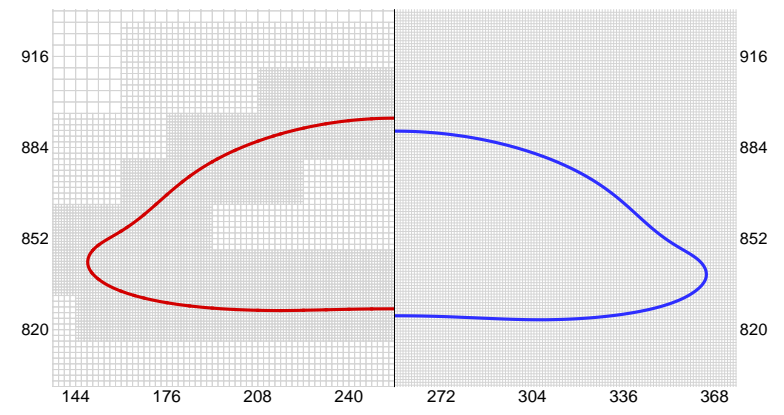

(b) $\mathrm{Eo}=20$

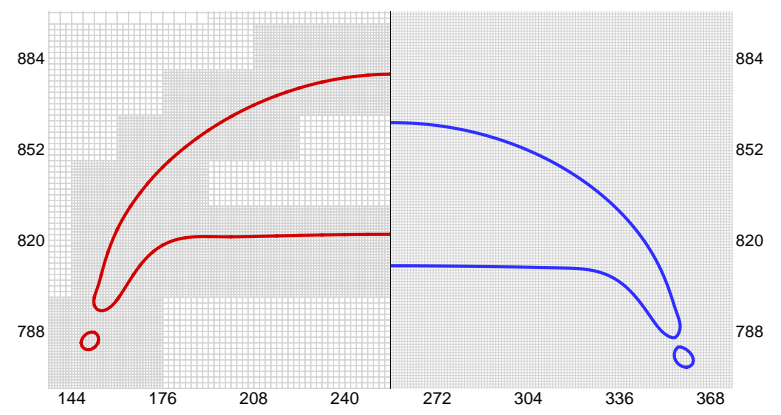

(c) $\mathrm{Eo}=200$

Figure 4: Bubble rising due to buoyancy force at $t^{*}=12$. Comparison between the AMR and the uniform-grid results. $\rho_{h} / \rho_{l}=1000, \mu_{h} / \mu_{l}=100$, and $\mathrm{Re}_{h}=100$. (a) Eo $=5$ $\left(\mathrm{Mo}=1.2 \times 10^{-6}\right) ;(\mathrm{b})$ Eo $=20\left(\mathrm{Mo}=8.0 \times 10^{-5}\right) ;(\mathrm{c})$ Eo $=200\left(\mathrm{Mo}=8.0 \times 10^{-2}\right)$. In each figure the left frame shows the AMR simulations with $l_{\max }=6$ (corresponding to a $512 \times 1536$ grid) and the right frame corresponds to the equivalent uniform-grid results. 


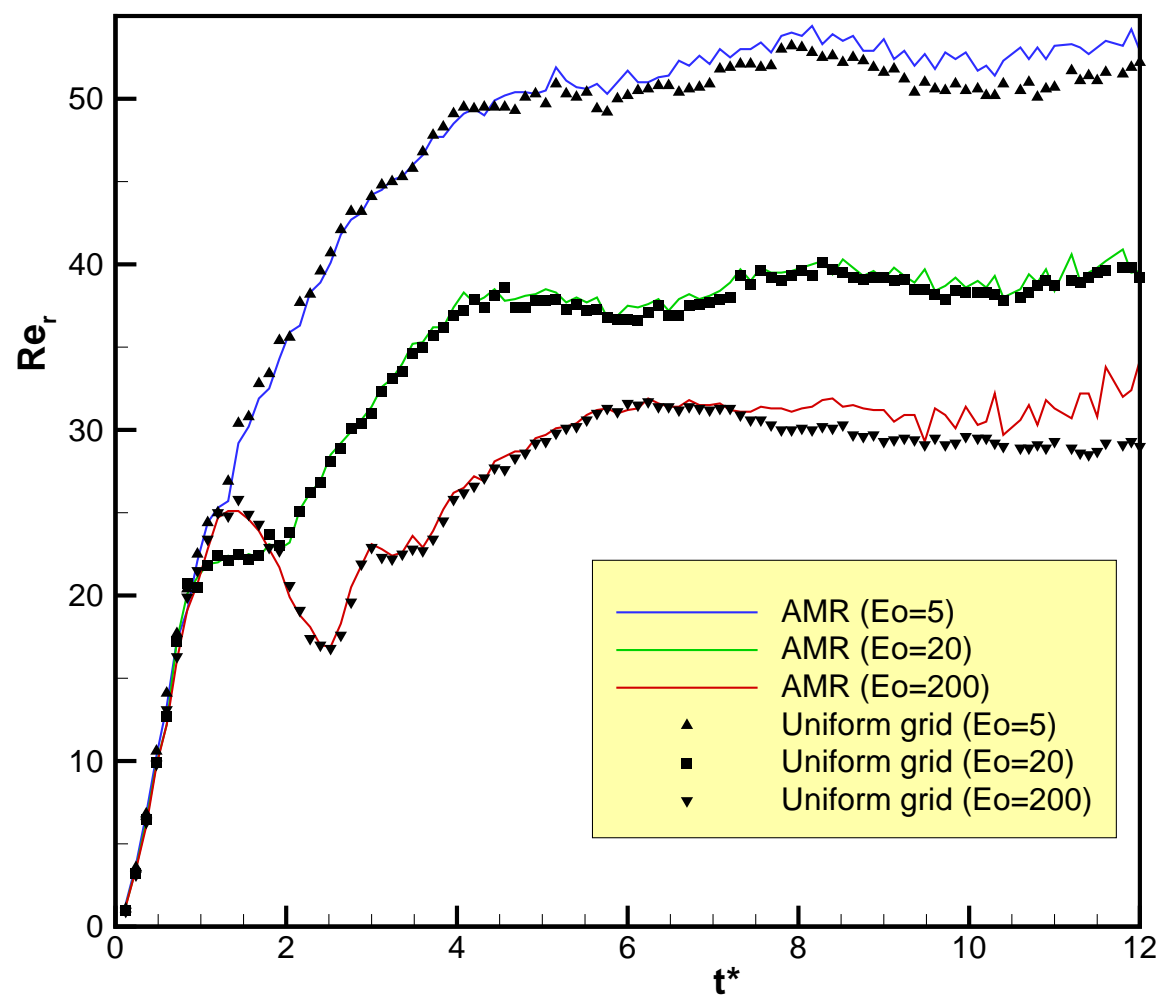

Figure 5: Evolution of the Reynolds number of the bubble as it rises. Comparison between the AMR and the uniform-grid solution.

\subsubsection{Efficiency}

Comparison between the performances of the current AMR-LBM and the equivalent uniform-grid code for rising bubble at Eo $=20$ is made in Table 1. The rising bubble simulations are carried out on an $\operatorname{Intel}(\mathrm{R}) \mathrm{Xeon}(\mathrm{R})$ workstation with CPU X5680 @3.33GHz and 12GB RAM. The GNU ForTRAN compiler with level 3 optimization is used to produce the data in Table 1. It is worth noting that in all of the simulations the refinement/derefinement subroutine is called at every time step. As it can be seen in Table 1, the 


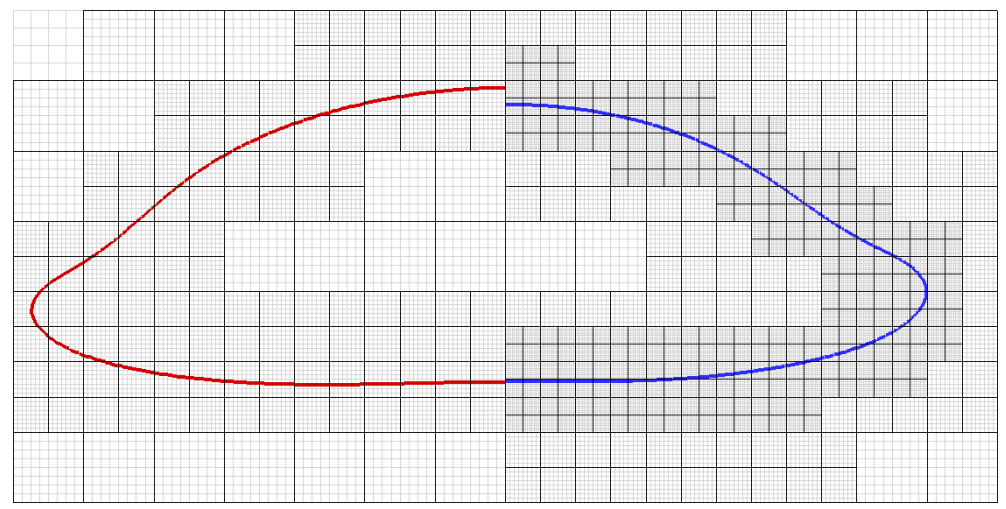

Figure 6: Bubble rising due to buoyancy force at $t^{*}=12$. Comparison between the AMR simulations at two different grid resolutions corresponding to different number of refinement levels. $\rho_{h} / \rho_{l}=1000, \mu_{h} / \mu_{l}=100, \operatorname{Re}_{h}=100$, and Eo $=20\left(\mathrm{Mo}=8.0 \times 10^{-5}\right)$. In the left frame $l_{\max }=6$ and in the right frame $l_{\max }=7$.

AMR-LBM code is about 18 times faster than the equivalent uniform-grid computations. The proposed AMR-LBM routine also consumes about 20 times less computational memory for storing the data. The actual saving in computational resources could be even higher when considering systems of larger sizes.

\subsubsection{Sensitivity study}

Before getting further into the simulations, we examine the effect of refinement criteria on the accuracy of the results by tunning $\epsilon_{r}$ and $\epsilon_{d}$. The AMR simulations of rising bubble are considered for $\rho_{h} / \rho_{l}=1000, \mu_{h} / \mu_{l}=100$, $\operatorname{Re}_{h}=100$, and Eo $=5\left(\mathrm{Mo}=1.2 \times 10^{-6}\right)$. The 2D ellipsoidal bubble

Table 1: CPU run time (in seconds) for rising bubble at Eo $=20$.

\begin{tabular}{|c|c|c|c|}
\hline Equivalent grid resolution & uniform-grid run time & AMR-LBM run time & speed-up factor \\
\hline $512 \times 1536$ & $50785(\mathrm{~s})$ & $2873(\mathrm{~s})$ & 18.2 \\
\hline
\end{tabular}




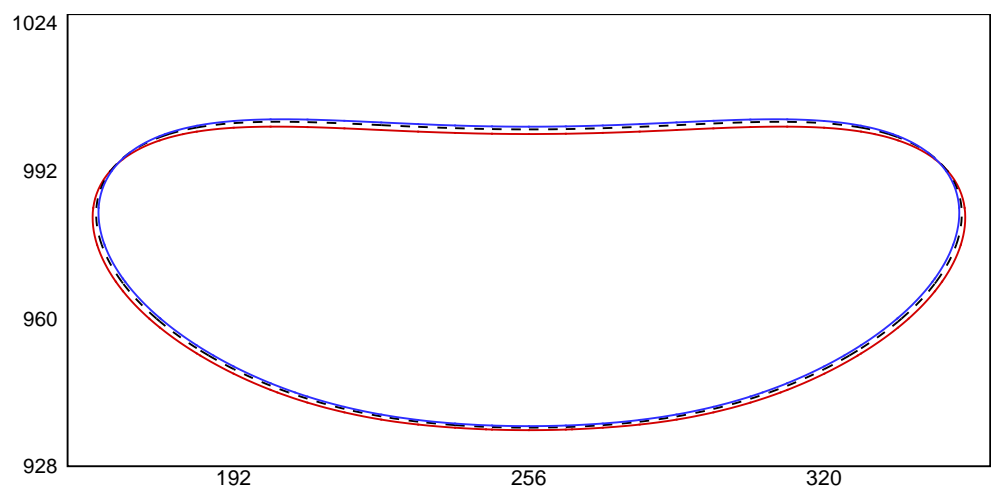

Figure 7: Sensitivity study for AMR-LBM simulations of rising bubble under buoyancy force at Eo $=5 . \epsilon_{r}=0.004$ (solid red line), $\epsilon_{r}=0.002$ (dashed black line), $\epsilon_{r}=0.001$ (solid blue line), and $\epsilon_{d}=\epsilon_{r} / 2$.

at $t^{*}=12$ is demonstrated in Fig. 7 for three different refinement criteria $\epsilon_{r}=0.004,0.002,0.001$ and $\epsilon_{d}=\epsilon_{r} / 2$. As it can be observed, qualitatively, the bubble shape remains almost the same when $\epsilon_{r}$ is changed from 0.004 to 0.001 . To put this in quantitative perspective, the relative difference in the terminal velocity of the bubble is less than $0.6 \%$ when changing the refinement criterion from $\epsilon_{r}=0.002$ to $\epsilon_{r}=0.001$.

\subsubsection{Mass conservation}

One of the key features of the proposed phase-field LBE is that it is mass conserving. The total mass of the system for the rising bubble is checked to verify the mass conservation property of the model, and the current results are compared with the previous model [21] on uniform grids. The total mass of the system $M_{t}$ is normalized by the initial mass $M_{0}$, and its evolution is plotted versus dimensionless time in Fig. 8. As it can be seen, for the method proposed in Ref. [21] the mass of the system gradually increases while the 


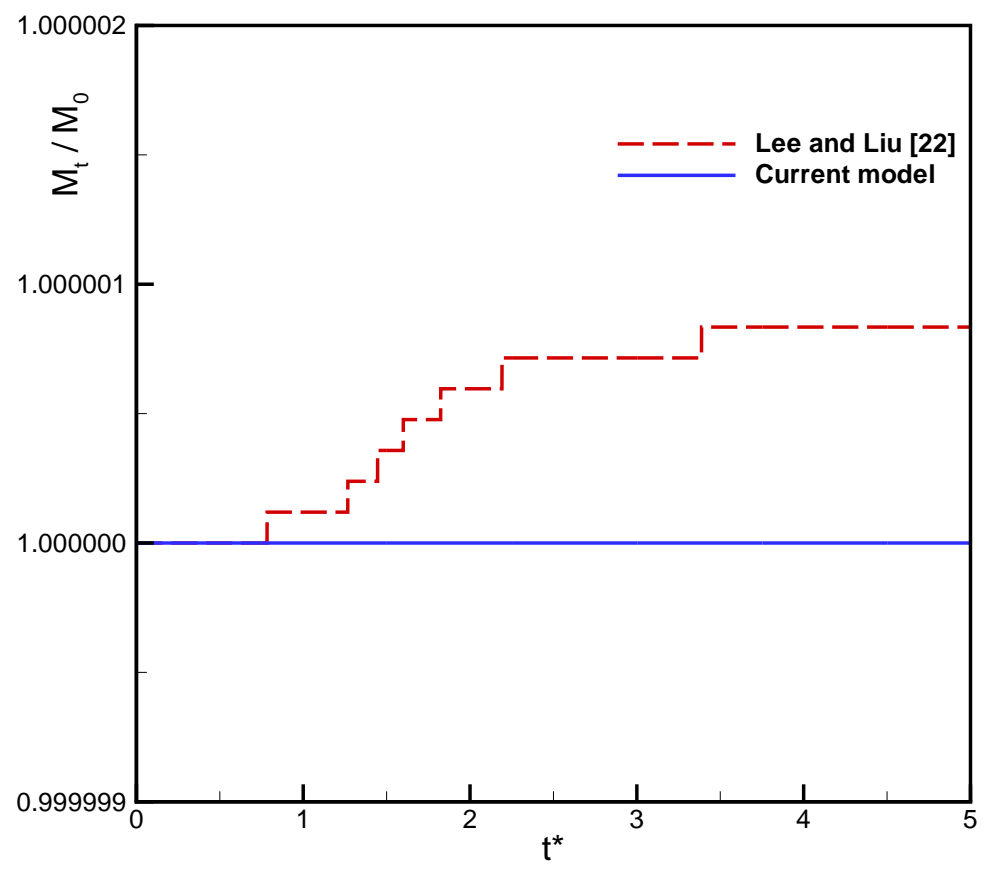

Figure 8: Variation of the mass of the system versus time for the rising bubble. The dashed red line shows the results of the model of Lee and Liu [21] and the solid blue line shows the results of the conservative phase-field model.

current model preserves the total mass.

\subsection{Falling droplet}

The dynamics of a falling drop under gravity is another interesting twophase flow phenomenon which has been studied previously [60-63]. Here, instead of a gas bubble surrounded by a heavy fluid, we place a liquid droplet with diameter $D=L_{0} / 5$ at $\left(L_{0} / 2,3 L_{0} / 2\right)$ and track its evolution as it falls due to the body force $\mathbf{F}_{b}=-\left(\rho-\rho_{l}\right) G_{y} \hat{\mathbf{j}}$. The computational domain and the boundary conditions are the same as in the rising bubble simulations. At a constant density ratio, the flow can be characterized by the Eötvös or the 
Bond number defined in Eq. (44) and the gravity Reynolds numbers based on the properties of light and heavy fluids

$$
\begin{aligned}
\operatorname{Re}_{l} & =\frac{\sqrt{G_{y} \rho_{l}\left(\rho_{h}-\rho_{l}\right) D^{3}}}{\mu_{l}} \\
\operatorname{Re}_{h} & =\frac{\sqrt{G_{y} \rho_{h}\left(\rho_{h}-\rho_{l}\right) D^{3}}}{\mu_{h}}
\end{aligned}
$$

In order to capture the breakup mechanisms of the falling drop, the simulations are conducted at a moderate density ratio, which also allows us to compare the current model with other LB models. Of particular interest is the original LB model proposed by Lee and Liu [21] (denoted by LL hereafter) and the kinetic-based model proposed by He, Chen, and Zhang [16] (denoted by HCZ hereafter). The former differs from the current LB model only in the interface tracking equation while the latter is significantly different from the current model with a distinctive difference in the underlying physics of the governing equations. In the HCZ model the Carnahan-Starling equation of state is used to calculate the thermodynamic pressure, which makes it essentially a single-component two-phase flow LB model. Moreover, the maximum and the minimum values of the index function and the surface tension force are not known a priori. They are determined either by constructing the Maxwell equal area principle or by using the flat-interface test.

Two sets of simulations are carried out at $\rho_{h} / \rho_{l}=10$ and Eo $=100$ with the numerical Péclet number $\mathrm{Pe}=2.0$ and the Cahn number $\mathrm{Cn}=$ 0.008. In the first case $\operatorname{Re}_{l}=20$ and $\operatorname{Re}_{h}=10$, and the evolution of the droplet as it falls due to the body force is depicted in Fig. 9 for different LB models. Generaly speaking, all LB models predict the onset of bag breakup at $t^{*}=9$ with some differences. The formation of a bag-shaped drop is 


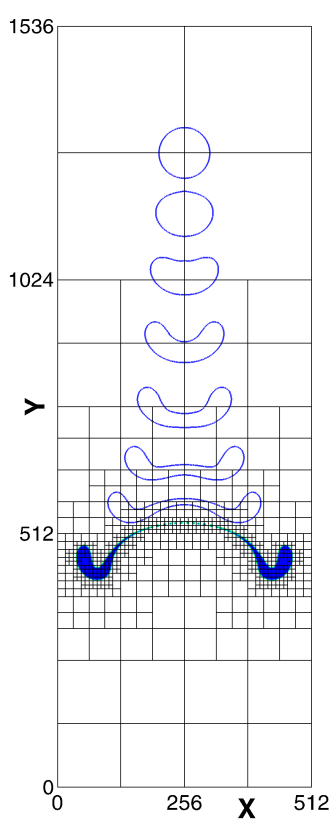

(a) AMR

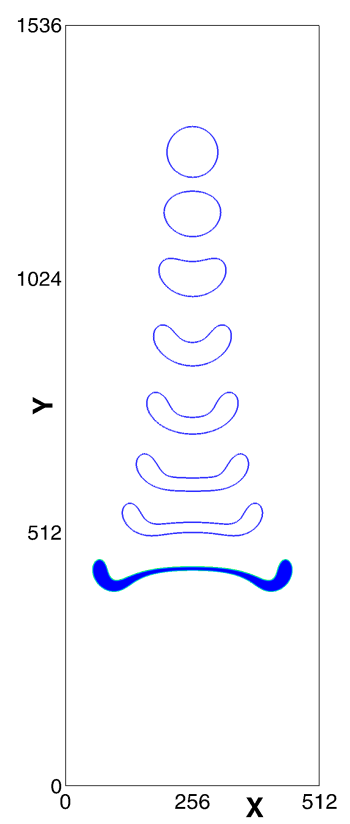

(b) Uniform grid

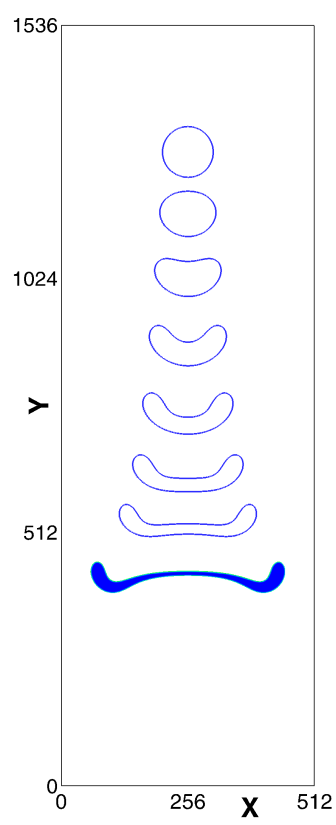

(c) LL

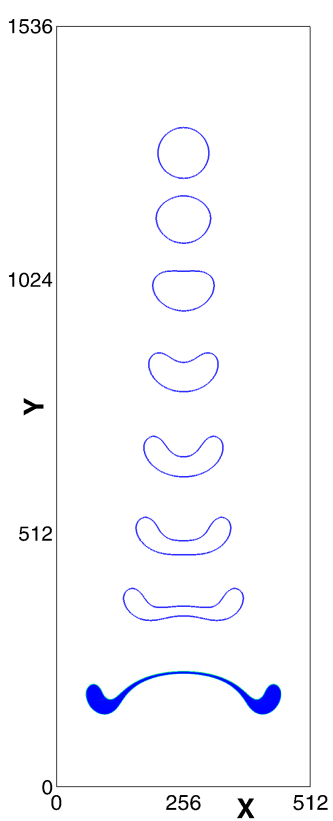

(d) $\mathrm{HCZ}$

Figure 9: Bag breakup of a falling droplet under the gravitational force. Comparison between the results of the (a) current AMR-LBM, (b) uniform grid, (c) model proposed by Lee and Liu [21], and (d) model proposed by He et al. [16]. $\rho_{h} / \rho_{l}=10$, Eo $=100$, $\operatorname{Re}_{l}=20$, and $\operatorname{Re}_{h}=10$. In each frame the last (flooded) contour corresponds to $t^{*}=9$.

more pronounced in the AMR-LBM result with some differences with the uniform-grid solution with respect to the depth of the bag. We suspect that either the Lax-Wendroff scheme or the interpolation polynomial could be the reason for this discrepancy. On the other hand, the uniform-grid results of the phase-field LBE agree well with the LL model. The drop in the HCZ model, however, travels more distance before it undergoes a bag-breakup mechanism. This type of breakup mechanism has previously been observed both in 2D [61] and in axisymmetric coordinates [62] using the HCZ model. 
In the second case the parameters are $\operatorname{Re}_{l}=15$ and $\mathrm{Re}_{h}=200$, and the results are shown in Fig. 10. Again, the general pattern of the results are similar in that all LB models predict a shear breakup mechanism. The quality of the drop breakup in the AMR and uniform-grid simulations are almost the same but this time the uniform-grid results of the conservative LBM and the LL model do not exactly match. As it is seen in Fig. 10, the phase-field LBE predicts thin, stretched ligaments of fluid that are attached to the falling drop at $t^{*}=9$ while the LL model shows disintegration of the ligament fluids into smaller satellite droplets. Also it is noted that the HCZ model predicts the shear breakup mechanism earlier at $t^{*}=8$ with some differences in the final shape of the fragmented drop.

In order to explain the discrepancy in the results, it is instructive to recall some of the differences between the HCZ model and the LL model. The HCZ model uses a Carnahan-Starling equation of state in the calculation of the pressure while the LL model invokes a chemical potential which is obtained by simplification of the van der Waals equation. The interface tracking equation of the LL model has shown to recover the Cahn-Hilliard equation with the numerical Péclet number $\mathrm{Pe}=\frac{U_{0} W}{M \beta}$ [21] while the interface tracking equation that is recovered from the HCZ model is not specified [16]. As such, we can adjust the mobility such that the Péclet number of the flow is unchanged when comparing our LB model with the LL model but there is no clear mobility value defined in the HCZ model to facilitate a transparent comparison. The above reasons may contribute to the minor differences observed in the breakup patterns of the falling drop in Figs. 9 and 10. Further investigation of the apparent discrepancies is beyond the scope of the present 


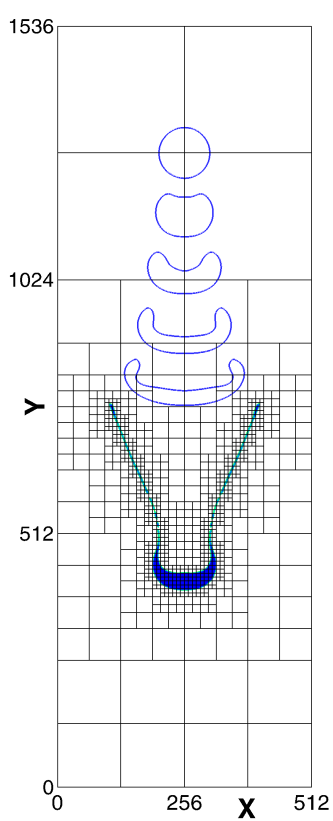

(a) AMR

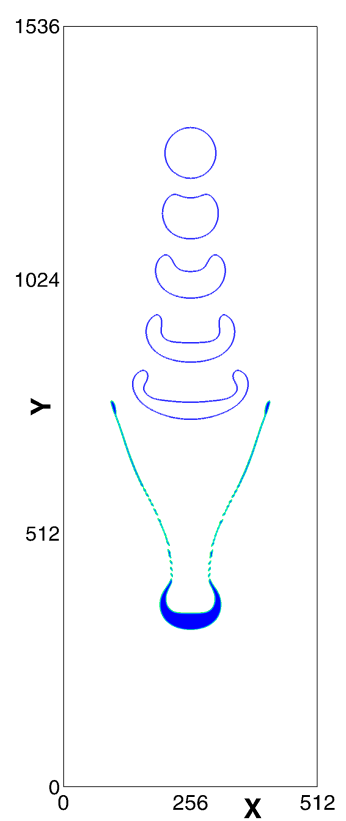

(b) Uniform grid

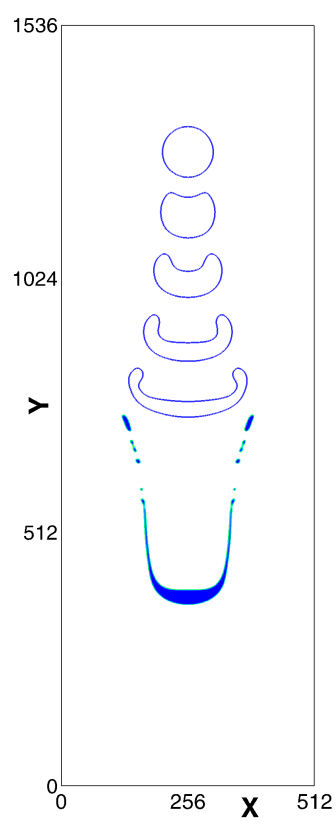

(c) LL

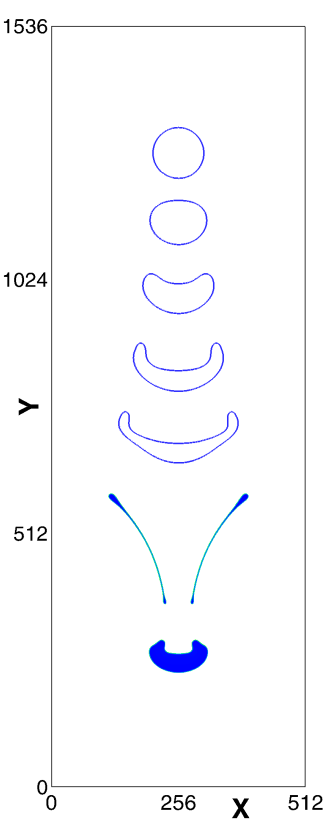

(d) $\mathrm{HCZ}$

Figure 10: Shear breakup of a falling droplet under the gravitational force. Comparison between the results of the (a) current AMR-LBM, (b) uniform grid, (c) model proposed by Lee and Liu [21], and (d) model proposed by He et al. [16]. $\rho_{h} / \rho_{l}=10$, Eo $=100$, $\operatorname{Re}_{l}=15$, and $\operatorname{Re}_{h}=200$. In each figure the last (flooded) contour corresponds to $t^{*}=9$ except for $(\mathrm{d})$ where $t^{*}=8$.

study and should be addressed elsewhere.

The grid resolution study is also performed within the AMR framework by increasing the maximum refinement level from $l_{\max }=6$ to $l_{\max }=7$. The numerical results are shown in Fig. 11 for two different cases; one at a fixed Cahn number $\mathrm{Cn}=0.008$ in Fig. 11(a) and one at a fixed interfacial width $W=4$ in Fig. 11(b). Although both cases show the bag breakup mechanism of the falling drop, the AMR results seem to be in better agreement with the results of the constant interfacial thickness case in Fig. 11(b). It is 


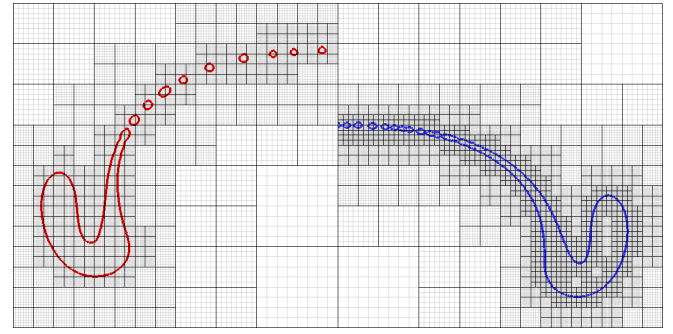

(a) $\mathrm{Cn}=0.008$

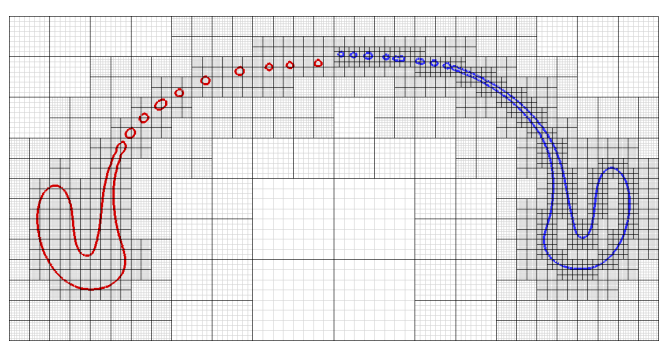

(b) $W=4$

Figure 11: Bag breakup of a falling droplet under the gravitational force at $t^{*}=10$. Comparison between the AMR simulations at two different grid resolutions corresponding to different number of refinement levels. $\rho_{h} / \rho_{l}=10$, Eo $=100, \operatorname{Re}_{l}=20$, and $\operatorname{Re}_{h}=10$. (a) The Cahn number is held fixed; (b) the interfacial thickness is kept constant. Within each figure in the left frame $l_{\max }=6$ and in the right frame $l_{\max }=7$.

also interesting to note that the current phase-field LBE is successful at capturing small satellite droplets without any difficulty, and in contrary to the Cahn-Hilliard-based interface tracking models [46] the satellite droplets with a small radius do not disappear easily. The convergence properties of diffuse-interface models are very well discussed by Magaletti et al. [56].

\subsubsection{Efficiency}

The efficiency of different LB solvers is also compared in Table 2. The falling drop simulations are carried out on an $\operatorname{Intel}(\mathrm{R}) \mathrm{Xeon}(\mathrm{R})$ workstation with CPU E5-26200 @2.00GHz and 32GB RAM. The GNU ForTran compiler with level 3 optimization is used to produce the data in Table 2. As it can be seen, the AMR-LBM code is about 23 times faster than the equivalent uniform-grid computations for the bag breakup simulations, and about 19 times faster for the shear breakup simulations. Among the LB solvers on uniform grids, the HCZ model is the most efficient one mainly because it 
avoids the calculation of mixed finite differences given in Eq. (30). On the downside, however, the HCZ model is restricted to relatively low density ratios. In Table 2 it is also seen that the current phase-field-based LBM is more efficient than the original LL model. This is due to the fact that the phasefield LBE for interface tracking is much simpler than the interface tracking model proposed by Lee and Liu [21]. The former only needs to calculate a 1st-order derivative (gradient of the phase field) while the latter requires the gradient of the pressure and the Laplacian of the chemical potential to be calculated. On top of that, only central differences are used in the former approach while both central and biased differences are involved in the LL model.

\subsection{Drop splashing on a wet surface}

Drop impact on a wet surface poses interesting dynamics and its simulation is challenging because in addition to large density and viscosity difference across the interface of the two fluids there exists a singularity at the impact point. Here we choose this problem to show the versatility of the proposed

Table 2: CPU run time (in seconds) for simulations of the falling droplet. Case 1 corresponds to the bag breakup mechanism in Fig. 9 and case 2 corresponds to the shear breakup mechanism in Fig. 10.

\begin{tabular}{|c|c|c|}
\hline LBM solver & CPU run time (case 1) & CPU run time (case 2) \\
\hline HCZ [16] & $167735(\mathrm{~s})$ & $168602(\mathrm{~s})$ \\
LL [21] & $299452(\mathrm{~s})$ & $300363(\mathrm{~s})$ \\
Current (uniform grid) & $249725(\mathrm{~s})$ & $250510(\mathrm{~s})$ \\
Current (AMR) & $10883(\mathrm{~s})$ & $13059(\mathrm{~s})$ \\
\hline
\end{tabular}


AMR-LBM model. The flow is characterized by two important dimensionless groups, namely Reynolds and Weber numbers

$$
\begin{gathered}
\operatorname{Re}=\frac{\rho_{h} U_{0} D}{\mu_{h}} \\
\mathrm{We}=\frac{\rho_{h} U_{0}^{2} D}{\sigma}
\end{gathered}
$$

where $U_{0}$ is the impact velocity of the drop. Here, the dimensionless time is

$$
t^{*}=\frac{t U_{0}}{D}
$$

According to the theoretical approximations, the spreading radius at initial stages follows the power-law relation

$$
r=\sqrt{D U_{0} t}
$$

We conduct the simulations for two sets of parameters at low and moderate Reynolds numbers corresponding to the deposition and splashing mechanisms. The density ratio and the Weber number are fixed at 1000 and 8000, respectively. In the first case $\operatorname{Re}=20\left(\mu_{h} / \mu_{l}=1000\right)$ while in the second case $\operatorname{Re}=100\left(\mu_{h} / \mu_{l}=200\right)$. The numerical Péclet number and the Cahn number are set to $\mathrm{Pe}=0.6$ and $\mathrm{Cn}=0.010$. The size of the computational domain is $3 L_{0} \times L_{0}$ and the drop is centered at $\left(3 L_{0} / 2, H+R\right)$ where $R=L_{0} / 5$ is the radius of the drop and $H=L_{0} / 10$ is the height of the liquid film. The boundary conditions are link bounce-back at the bottom and top of the domain and periodic in the $x$-direction. The deposition and splashing states shown in Fig. 12 are in accordance with the previous findings by Lee and Lin [18]. To further verify our observations, the current AMR-LBM results are compared with both the uniform-grid solution and the AMR results 
at a higher resolution. The comparison is shown in Fig. 13 for the splashing state at $R e=100$. It can be observed that the AMR results are in satisfactory agreement with the uniform-grid solution. Also the grid independency of the results is confirmed at a constant Cahn number $(\mathrm{Cn}=0.01)$.

The dimensionless spread radius $r / D$ versus the dimensionless time is also plotted in Fig. 14. Although the numerical spreading factor is overestimated by our numerical results, it obeys a power-law curve as predicted by analytical approximations. The main reason for the discrepancy is that our simulations are in $2 \mathrm{D}$.

\subsection{Droplet coalescence onto a fluid interface}

A droplet placed in the vicinity of a flat interface can exhibit different behaviors depending on the overriding factors characterized by gravitational forces, viscous stress, and capillary pressure [64-66]. In addition to the density and viscosity contrasts, the important dimensionless groups are the Bond number defined in Eq. (44) (Bo= Eo), and the Ohnesorge number

$$
\mathrm{Oh}=\frac{\mu_{h}}{\sqrt{\rho_{h} D \sigma}}
$$

The dimensionless time is $t^{*}=t / t_{c}$, where the capillary time is defined by

$$
t_{c}=\sqrt{\frac{\rho_{h} D^{3}}{\sigma}}
$$

From the diffuse-interface modeling point of view, it is numerically very challenging to capture the time period where the droplet is standing still on top of the flat interface while the interface deforms due to very strong capillary pressure forces stemming from the lubrication effects [67, 68]. Intrinsic to the diffuse-interface models, the droplet rapidly merges onto the interface. 


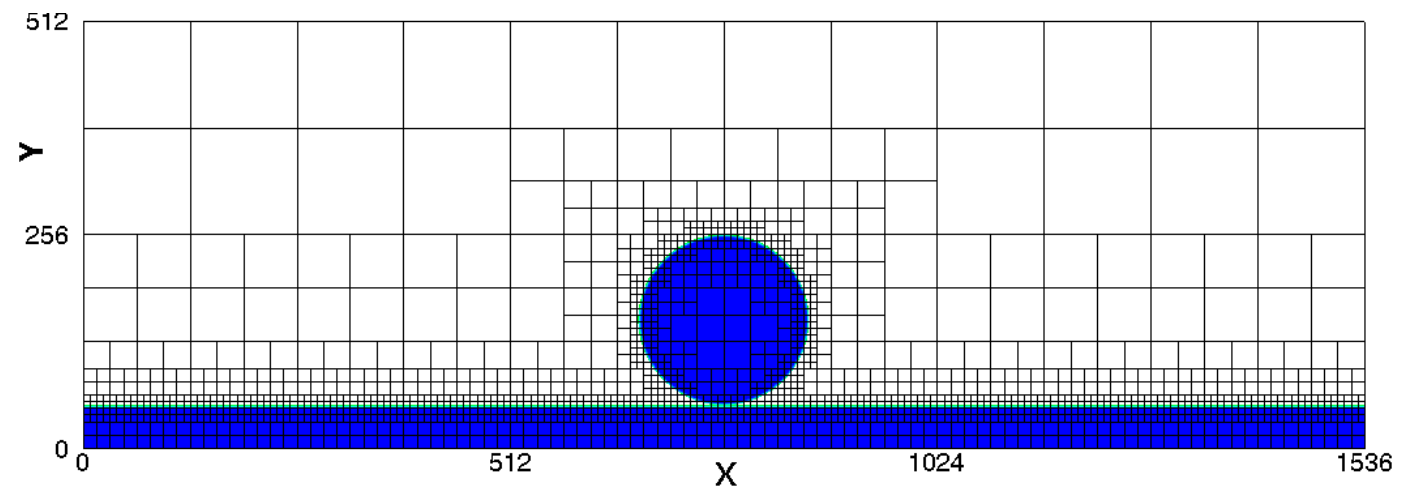

(a) Initial profile $\left(t^{*}=0.0\right)$

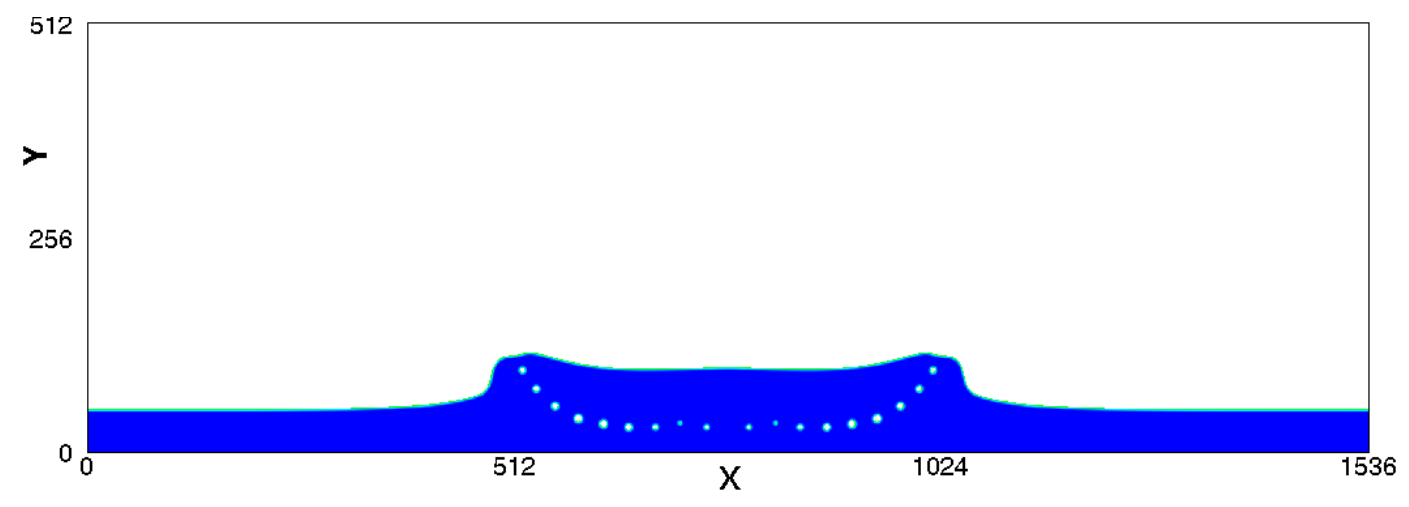

(b) $\operatorname{Re}=20\left(t^{*}=1.6\right)$

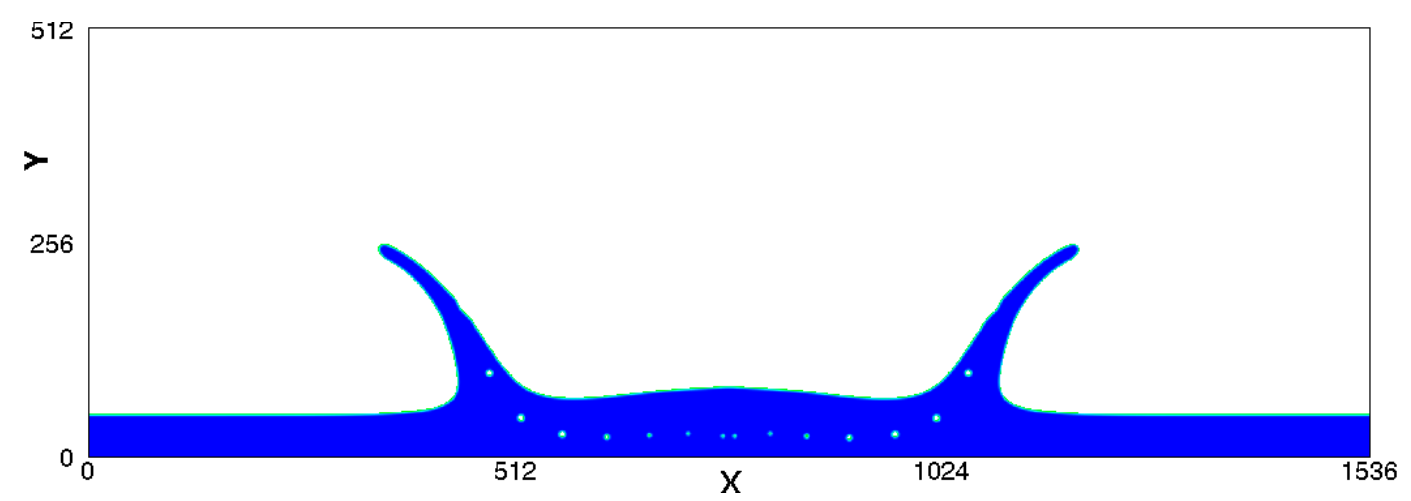

(c) $\operatorname{Re}=100\left(t^{*}=1.6\right)$

Figure 12: Drop impact on a wet surface. $\rho_{h} / \rho_{l}=1000$ and $\mathrm{We}=8000$. (a) Initial profile $\left(t^{*}=0.0\right)$; (b) deposition state at $\operatorname{Re}=20\left(t^{*}=1.6\right) ;(\mathrm{c})$ splashing state at $\operatorname{Re}=100$ $\left(t^{*}=1.6\right)$. 


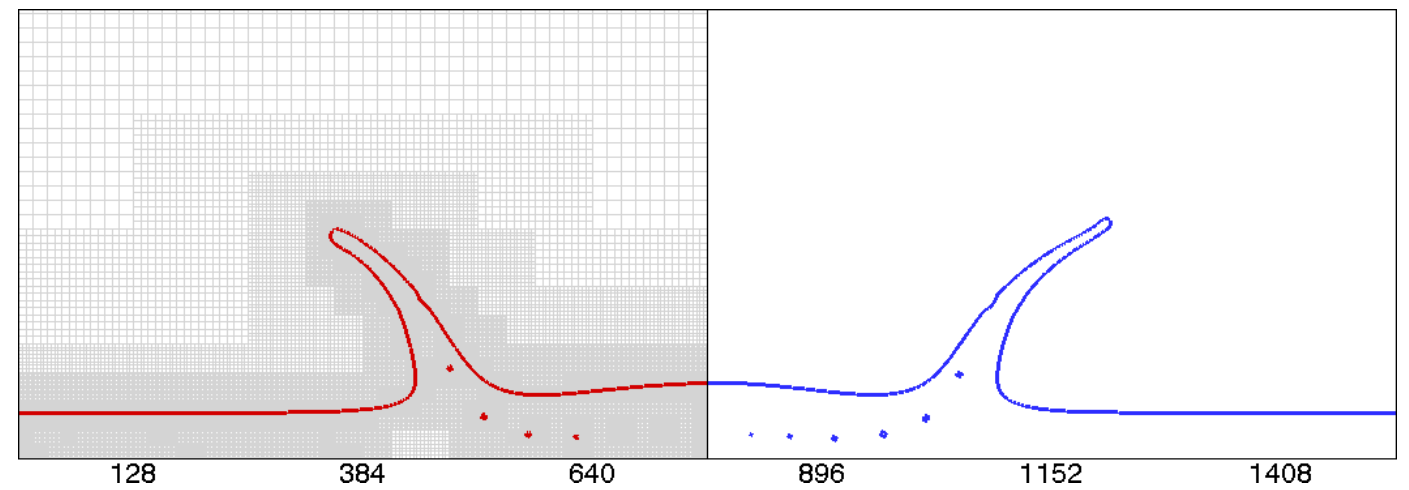

(a)

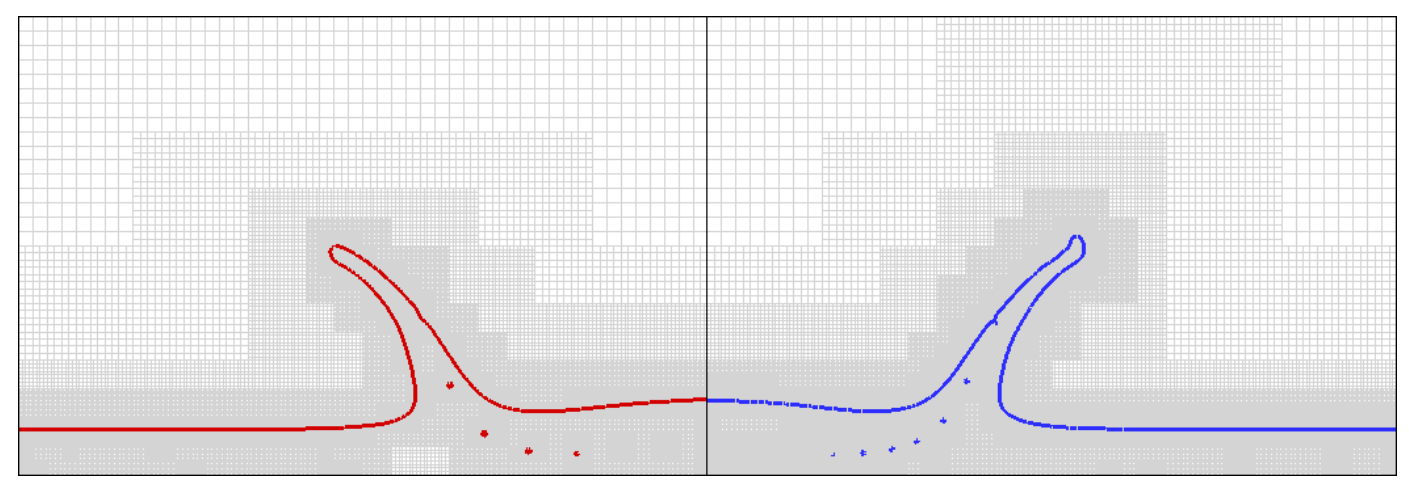

(b)

Figure 13: Droplet splashing on a wet surface at $t^{*}=1.6 . \rho_{h} / \rho_{l}=1000, \mathrm{We}=8000$, and $\operatorname{Re}=100\left(\mu_{h} / \mu_{l}=200\right)$. (a) Left: AMR-LBM results, right: uniform-grid solution; (b) AMR-LBM results with $l_{\max }=6$ (left) and $l_{\max }=7$ (right).

This, of course, depends on a number of factors such as the mobility, interface thickness, and the gap between the droplet and the fluid bath. Here, we consider the effects of mobility and interfacial thickness on the quality of droplet coalescence onto the flat interface.

Following previous studies $[64,66]$, we choose $\rho_{h} / \rho_{l}=1.2, \mu_{h} / \mu_{l}=1$, $\mathrm{Bo}=0.8$, and $\mathrm{Oh}=0.08$. The Cahn number is also fixed at $\mathrm{Cn}=0.006$ 


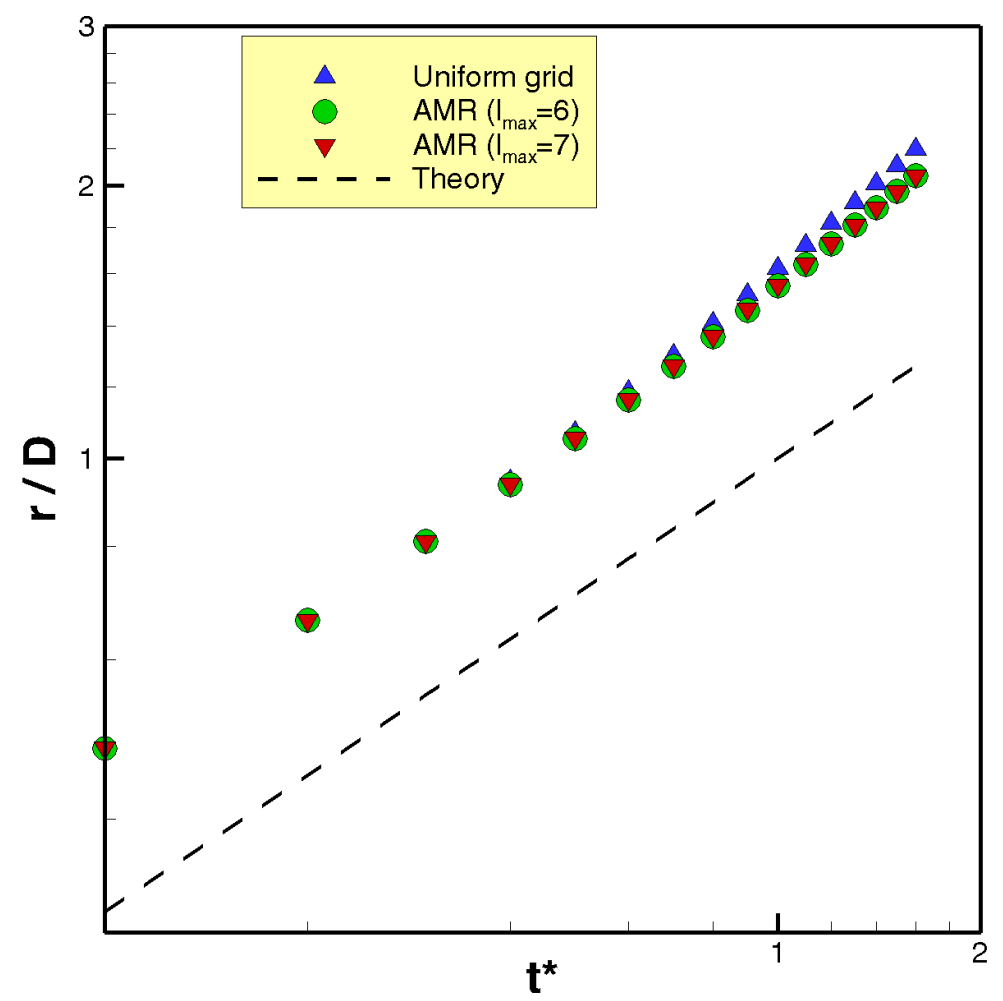

Figure 14: Theoretical and numerical results of spreading radius at initial stages of drop splashing on a wet surface. $\rho_{h} / \rho_{l}=1000, \mathrm{We}=8000$, and $\operatorname{Re}=100\left(\mu_{h} / \mu_{l}=200\right)$.

( $W=3$ ). Initially, a droplet with radius $R=L_{0} / 8$ is placed at $\left(L_{0} / 2, H+\right.$ $R+\delta$ ) inside a computational domain of size $L_{0} \times L_{0}$, where $H=L_{0} / 4$ is the height of the fluid bath beneath the droplet and $\delta$ is the gap between the drop interface and the fluid bath. Following the guidelines in previous studies $[64,66]$, we initially set $\delta=5$. The boundary conditions are link bounce-back at the bottom and top of the domain and periodic in the $x$ direction. The initial conditions are approximated by a hydrostatic pressure field with a constant body force $\mathbf{F}_{b}=-\rho G_{y} \hat{\mathbf{j}}$ throughout the domain.

Figure 15 shows the evolution of the droplet with $M=0.01(\mathrm{Pe}=10.6)$. 


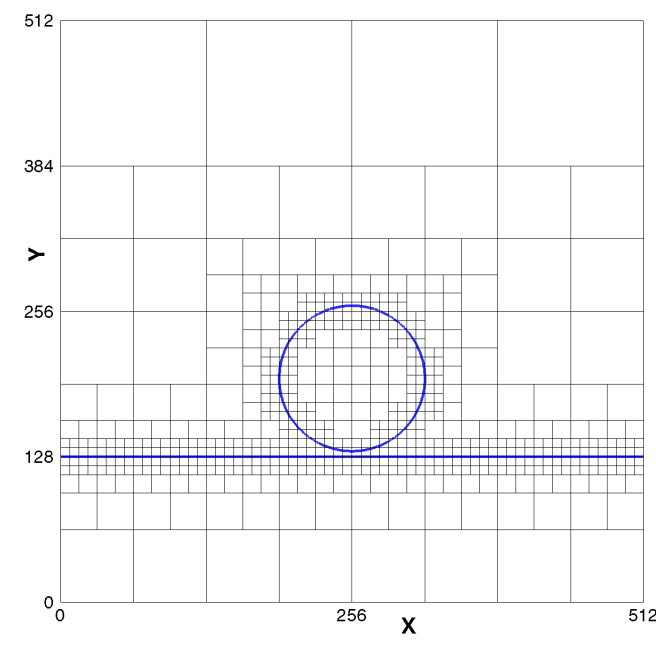

(a) $t^{*}=0.0$

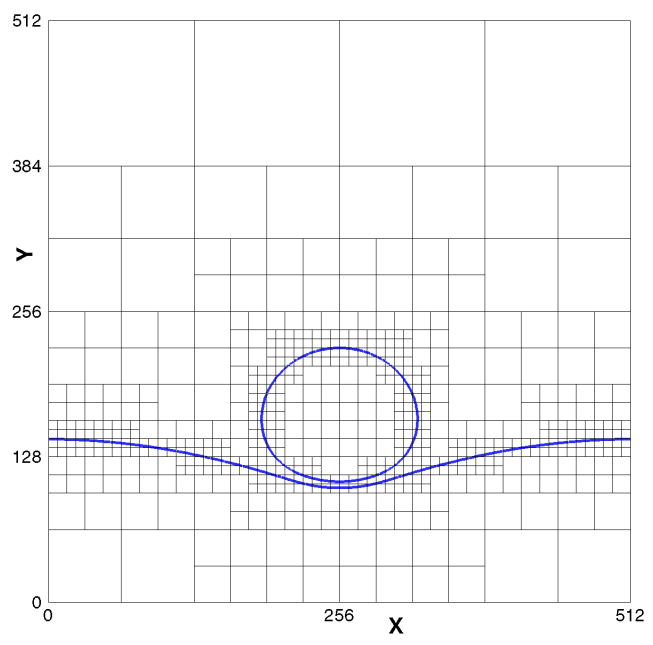

(b) $t^{*}=3.0$

Figure 15: A single droplet under buoyancy force sitting on top of a flat interface. $\rho_{h} / \rho_{l}=$ $1.2, \mu_{h} / \mu_{l}=1, \mathrm{Bo}=0.8, \mathrm{Oh}=0.08, \mathrm{Cn}=0.006$, and $M=0.01(\mathrm{Pe}=10.6)$. (a) $t^{*}=0.0$; (b) $t^{*}=3.0$.

We intentionally chose a relatively low value for the mobility to capture the moment that the droplet sits steadily on top of the deformed interface. Increasing the mobility expedites the droplet mergence onto the interface. A closeup of the displacement between the droplet and the deformed interface is provided in Fig. 16 for $M=0.015(\mathrm{Pe}=7.1)$ at different times.

The effect of the mobility on the instant of coalescence between the droplet and the fluid bath is shown in Fig. 17. As expected, the droplet merges onto the fluid bath more quickly at higher mobility values. In our simulations we found that increasing the interfacial thickness also expedites the coalescence. 


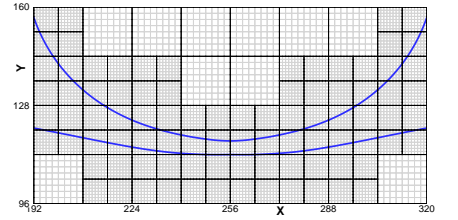

(a) $t^{*}=1.40$

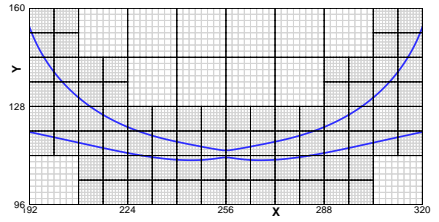

(b) $t^{*}=1.52$

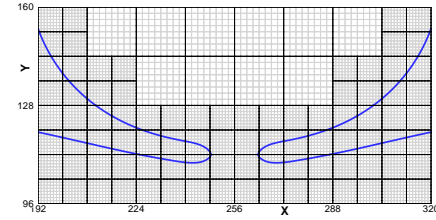

(c) $t^{*}=1.56$

Figure 16: Droplet coalescence onto a flat interface. $\rho_{h} / \rho_{l}=1.2, \mu_{h} / \mu_{l}=1$, Bo $=0.8$, $\mathrm{Oh}=0.08, \mathrm{Cn}=0.006$, and $M=0.015(\mathrm{Pe}=7.1):(\mathrm{a}) t^{*}=1.40 ;(\mathrm{b}) t^{*}=1.52 ;(\mathrm{c})$ $t^{*}=1.56$

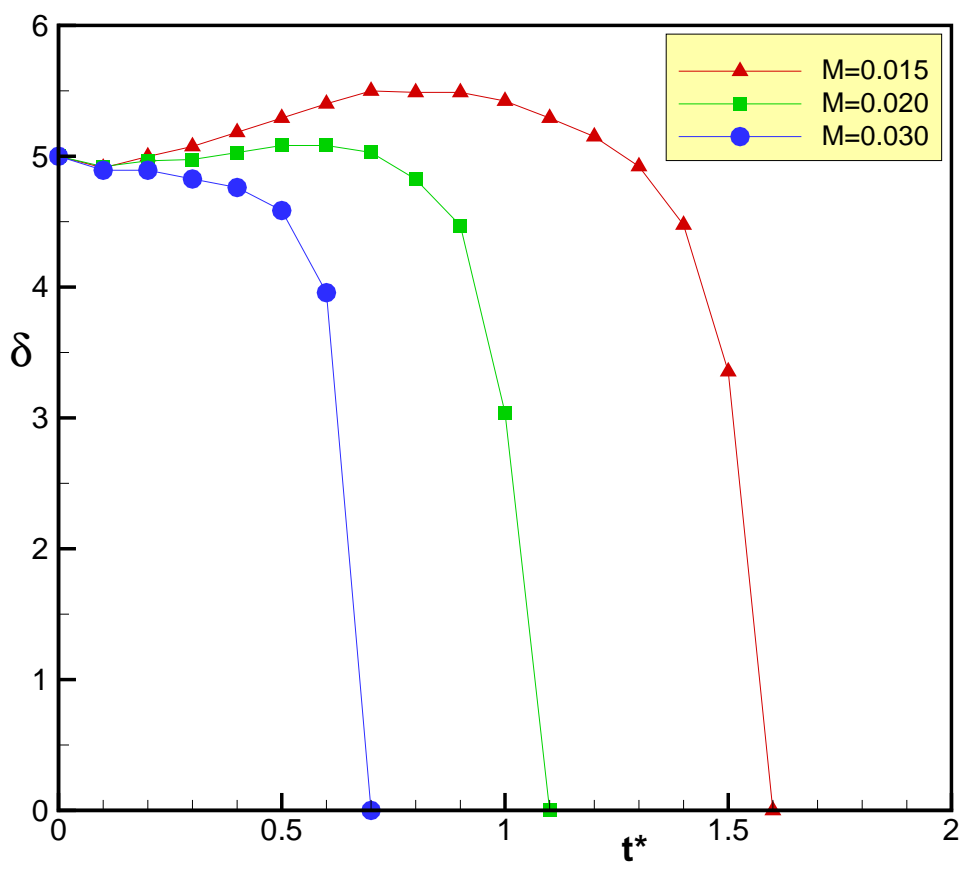

Figure 17: Dimensionless coalescence time versus displacement between the interface of the droplet and the fluid bath for different mobility values. $\rho_{h} / \rho_{l}=1.2, \mu_{h} / \mu_{l}=1$, $\mathrm{Bo}=0.8, \mathrm{Oh}=0.08$, and $\mathrm{Cn}=0.006$. 


\section{Kelvin-Helmholtz instability of a shear-layer flow}

In a recent study [22], we examined the Kelvin-Helmholtz instability (KHI) of a stratified shear layer $[69,70]$ for both single-phase and multiphase flows on uniform grids. We have also examined the single-phase KHI on nonuniform grids using the AMR-LBM [30,40] and obtained reasonably accurate results. In this section, we consider the KHI of a two-phase shearlayer flow for both density-matched binary fluids and two immiscible fluids with a density contrast using the current AMR-LBM. The results are presented in terms of four dimensionless groups: the density ratio $\rho_{h} / \rho_{l}$, the viscosity ratio $\mu_{h} / \mu_{l}$, the Weber number

$$
\mathrm{We}=\frac{\rho_{l} U^{2} L_{0}}{\sigma}
$$

and the Reynolds number

$$
\operatorname{Re}=\frac{\rho_{l} U L_{0}}{\mu_{l}}
$$

where $U=2 U_{0}$ is the characteristic velocity reference and the dimensionless

time is $t^{*}=t U / L_{0}$. The size of the computational domain is $L_{0} \times 2 L_{0}$ and the interface location at $t^{*}=0$ is explicitly given by the following dimensionless equation $[69,70]$

$$
\phi(x, y)=\frac{y}{L_{0}}+0.01 \sin \left(\frac{2 \pi(x+y)}{L_{0}}\right)
$$

Initially, a uniformly concentrated vorticity distribution in the form of the Dirac $\delta$ function is prescribed in the entire fluid

$$
\omega_{0}(x, y)=\delta_{h}(\phi)
$$


The following smoothed cosine function is used to approximate the above Dirac $\delta$ function [71]:

$$
\delta_{h}(\phi)=\left\{\begin{array}{lr}
\frac{\pi+2 \sin (\pi(2 \phi+1) / 4)-2 \sin (\pi(2 \phi-1) / 4)}{4 \pi}, & |\phi|<1.5 \\
\frac{5 \pi-2 \pi|\phi|-4 \sin (\pi(2|\phi|-1) / 4)}{8 \pi}, & 1.5 \leq|\phi| \leq 2.5 \\
0, & |\phi|>2.5
\end{array}\right.
$$

Given the vorticity distribution in the domain, we can solve the Poisson equation for the stream function

$$
\nabla^{2} \psi=-\omega_{0}=-\delta_{h}(\phi)
$$

and find the velocity distribution in the domain via $\mathbf{u}=\nabla \times \psi$, where the boundary conditions are $u_{x}(x, 0)=U_{0}$ and $u_{x}\left(x, 2 L_{0}\right)=-U_{0}$. In order to minimize the compressibility effects, we choose the Mach number of the flow such that $\mathrm{Ma}=U_{0} / c_{s}<0.05$.

\subsection{Density-matched fluids}

First, the density-matched immiscible fluids are considered at $\operatorname{Re}=20000$ with $\mathrm{Pe}=15$ and $\mathrm{Cn}=0.006$. The initial configuration of the AMR blocks together with the interface location and the vorticity contours for two different Weber numbers are shown in Fig. 18. As it can be seen in Fig. 18(b), a symmetric interface roll-up is created for $\mathrm{We}=200$ at $t^{*}=3.0$ while at a low enough Weber number in Fig. 18(c) fingers of interpenetrating fluids are formed. This behavior is expected because at smaller Weber numbers the surface tension force is stronger and therefore the interface tends to be more stable. Our results are in line with the previous observations [69, 70] and agree well with the findings by Ceniceros and Roma [70]. 


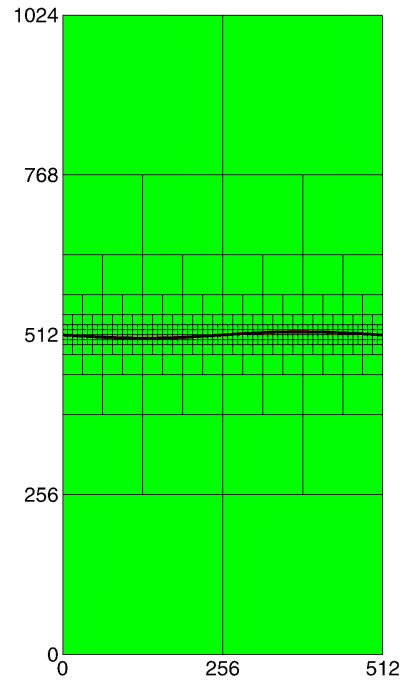

(a) Initial profile

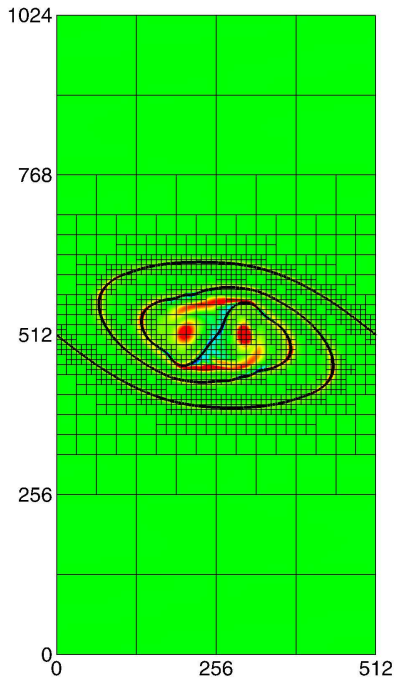

(b) $\mathrm{We}=200$

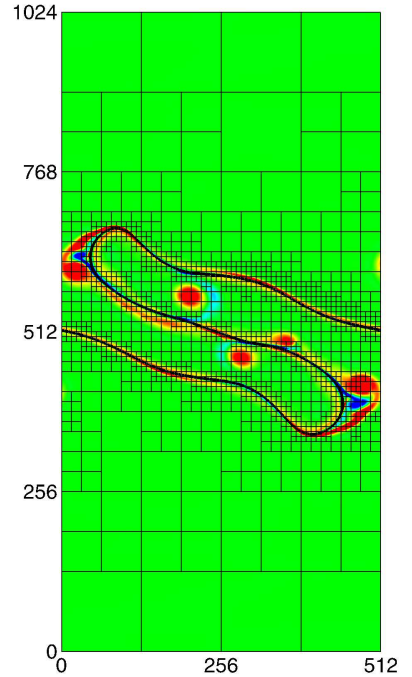

(c) $\mathrm{We}=50$

Figure 18: Vorticity (flooded contour) and interface location (black line) for binary fluids in a shear flow. $\rho_{h} / \rho_{l}=1, \mu_{h} / \mu_{l}=1$, and $\operatorname{Re}=20000$. (a) Initial configuration $\left(t^{*}=0\right)$; (b) $\mathrm{We}=200\left(t^{*}=3.0\right)$; (c) $\mathrm{We}=50\left(t^{*}=3.0\right)$.

For further evaluation of the current model, the AMR simulations are performed for two other sets of parameters and the findings are compared with the AMR results of the Lagrangian-marker model [70] in Fig. 19. As it can be seen, qualitatively, the results are in good agreement with the benchmark study.

\subsection{Density-stratified fluids}

Noting that the majority of available studies are focused on two miscible fluids with the Boussinesq approximation or immiscible density-matched binary fluids, we consider the effect of density ratio on the interface evolution. We examined three different density ratios at $\mathrm{Re}=10000$ with $\mathrm{Pe}=30$ and $\mathrm{Cn}=0.010$. The numerical results are shown in Fig. 20 and Fig. 21 

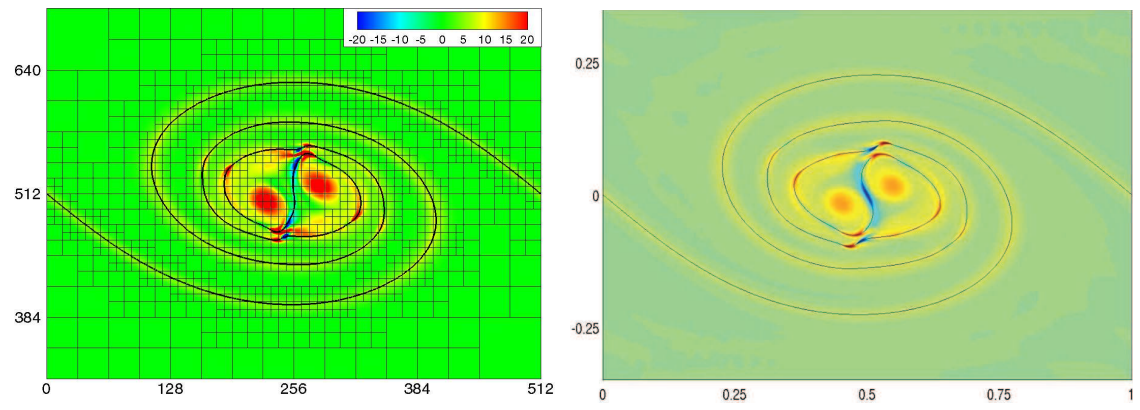

(a) $\mathrm{Re}=10000, \mathrm{We}=400$
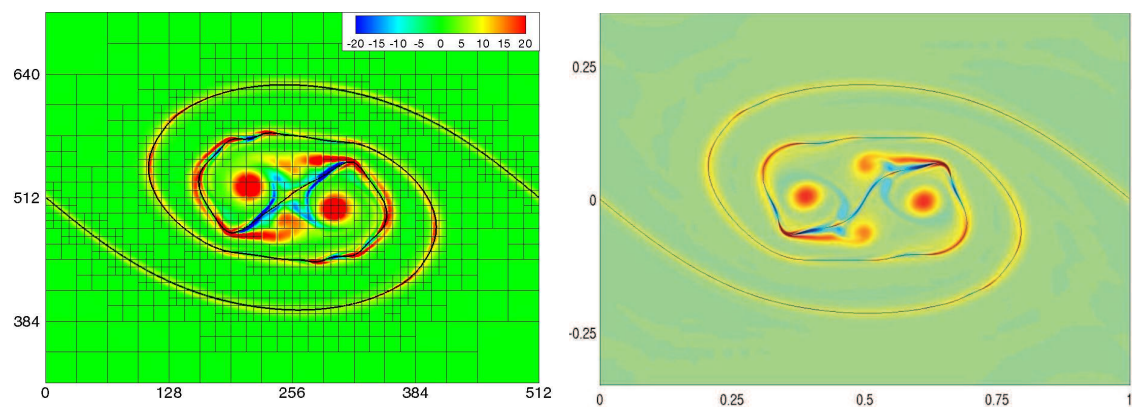

(b) $\mathrm{Re}=20000, \mathrm{We}=200$

Figure 19: Vorticity (flooded contour) and interface location (black line) for binary fluids in a shear flow at $t^{*}=2.5 . \rho_{h} / \rho_{l}=1$ and $\mu_{h} / \mu_{l}=1$. (a) $\operatorname{Re}=10000$ and $\mathrm{We}=400$; (b) $\operatorname{Re}=20000$ and We $=200$. Left: current AMR-LBM; right: benchmark study [70].

for two different Weber numbers. As it can be seen, increasing the density ratio results in severe topological changes at the interface and transition to turbulence in the flow field. The chaotic evolution of the interface at higher density ratios is more pronounced at higher Weber numbers in Fig. 21. This is due to the fact that at higher density ratios the inertial force of the heavy fluid dominates the viscous forces causing a highly deformed interface and eventually catastrophic breakup of the interface. Also, it is noticed that the flow field is no longer symmetric for the fluids with different densities. This 


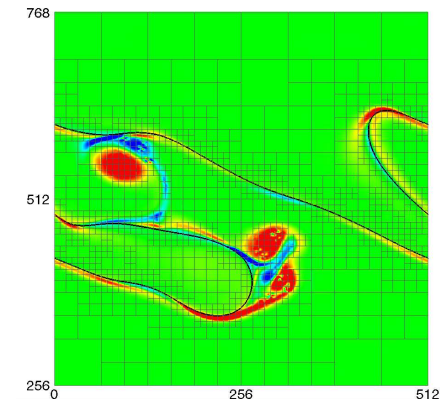

(a) $\frac{\rho_{h}}{\rho_{l}}=2$

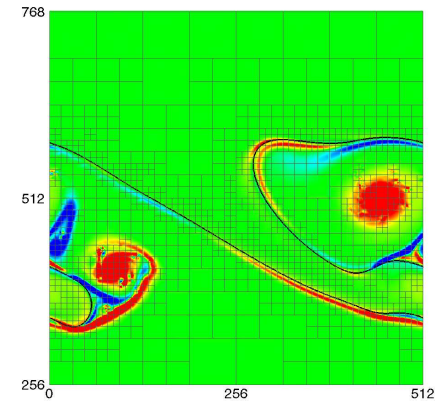

(b) $\frac{\rho_{h}}{\rho_{l}}=5$

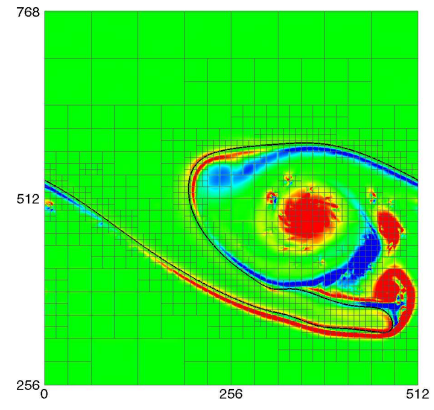

(c) $\frac{\rho_{h}}{\rho_{l}}=10$

Figure 20: Vorticity (flooded contour) and interface location (black line) of a stratified fluid in a shear-layer flow at $t^{*}=3.0 . \quad \mu_{h} / \mu_{l}=10, \mathrm{Re}=10000$, and $\mathrm{We}=50$. (a) $\rho_{h} / \rho_{l}=2$; (b) $\rho_{h} / \rho_{l}=5$; (c) $\rho_{h} / \rho_{l}=10$.

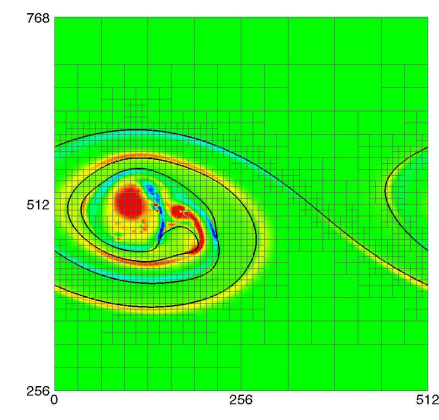

(a) $\frac{\rho_{h}}{\rho_{l}}=2$

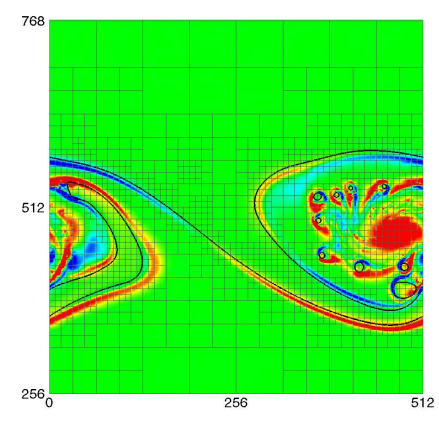

(b) $\frac{\rho_{h}}{\rho_{l}}=5$

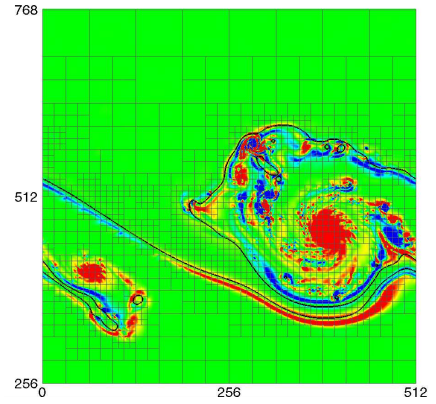

(c) $\frac{\rho_{h}}{\rho_{l}}=10$

Figure 21: Vorticity (flooded contour) and interface location (black line) of a stratified fluid in a shear-layer flow at $t^{*}=3.0 . \quad \mu_{h} / \mu_{l}=10, \mathrm{Re}=10000$, and $\mathrm{We}=400$. (a) $\rho_{h} / \rho_{l}=2 ;$ (b) $\rho_{h} / \rho_{l}=5$; (c) $\rho_{h} / \rho_{l}=10$.

interface asymmetry was also observed by Tauber et al. [69].

Formation of turbulent patterns in the flow field for $\rho_{h} / \rho_{l}=10$ is more evident in Fig. 22. As it can be seen, small Kelvin-Helmholtz wavelets are created at initial times, which trigger the transition to the chaotic flow field 


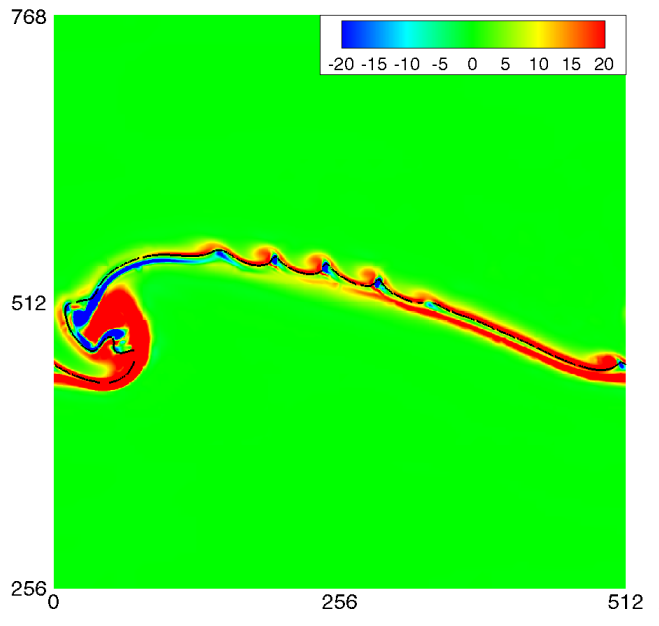

(a) $t^{*}=1.2$

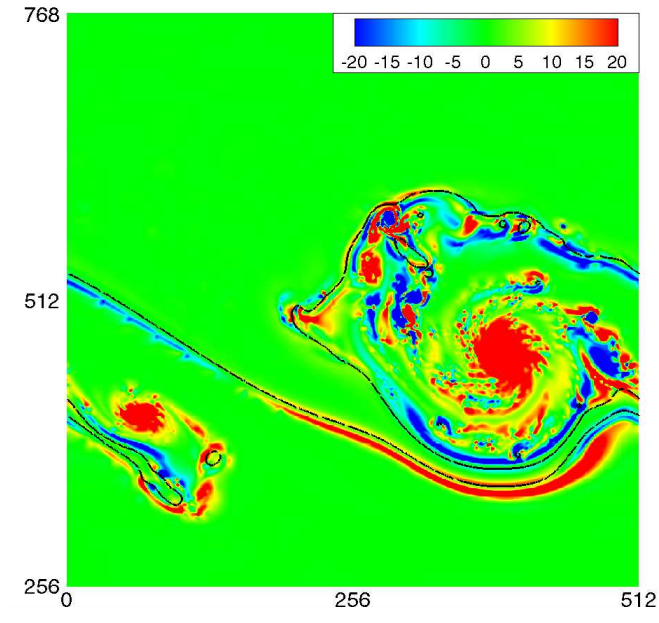

(b) $t^{*}=3.0$

Figure 22: Vorticity (flooded contour) and interface location (black line) of a stratified fluid in a shear-layer flow. $\rho_{h} / \rho_{l}=10, \mu_{h} / \mu_{l}=10, \operatorname{Re}=10000$, and $\mathrm{We}=400$. (a) $t^{*}=1.2 ;(\mathrm{b}) t^{*}=3.0$.

at later times. The catastrophic disintegration of the interface at $t^{*}=3.0$ is well captured and the small scale billows are adequately resolved by the current AMR-MRT-LBM. The very basic interface roll-up and the chaotic interface evolution are typical mechanisms observed in laminar and turbulent jets $[72-74]$.

\section{Summary and conclusion}

Based on the conservative phase-field model for interface tracking and the pressure evolution equation for recovering the hydrodynamic properties, an MRT-LBM for two-phase flows was developed. The proposed MRT-LBM was also reformulated on nonuniform grids and equipped with an AMR tech-

nique. It was shown, unlike the previous model [21], that the mass of the 
fluid is conserved and the computations are performed more efficiently by reducing the required memory and enhancing the speed-up. The locality of the collision step has also increased significantly due to multiple reasons; first, the phase-field LBE for interface tracking requires the calculation of only one derivative (the gradient of the phase field). It is also possible to implement an entirely local collision by invoking central moments to calculate the interface normal [49]. Although, for accuracy and stability reasons, we favored the use of isotropic finite-difference schemes over central moments to calculate the gradient of the phase field, the resulting method maintains its efficiency because the derivatives of the phase-field variable appear in the pressure evolution equation, for example in the calculation of the surface tension force. On the other hand, while the gradients of the pressure, density, and chemical potential appear in the original formulation of the two-phase LBM [21], here there is no need to transfer a portion of the date for $p, \rho$, or $\mu_{\phi}$ between adjacent blocks. The pressure gradient no longer exists in the present phase-field LBE; the gradient of $\rho$ is eliminated by Eq. (25); and $-\phi \nabla \mu_{\phi}$ is replaced with an equivalent surface tension force $\mu_{\phi} \nabla \phi$.

A variety of benchmark studies were carried out to examine the accuracy and reliability of the proposed model. The grid independency of the findings was examined by comparing the AMR-LBM results with the uniform-grid solution and also by increasing the number of refinement levels. The numerical model was further validated by comparing the simulations with other benchmark studies and other LB solvers. Different aspects of multiphase flow phenomena such as rapid topological changes, breakup mechanism, coalescence, and transition to turbulent flow were successfully simulated. In essence, the 
proposed AMR-MRT-LBM proved to be a promising tool for numerical simulation of two-phase flows at high Reynolds numbers. However, the current model cannot handle both large density difference and high-Reynolds-number flow simultaneously. Proposing a mass conserving two-phase LBM that can concurrently tackle both large density ratio and high Reynolds number is a demanding topic left for future work.

Although the current AMR-LBM was developed in 2D, its extension to $3 \mathrm{D}$ is straightforward and is a work in progress. The D2Q9 lattice can simply be replaced with the D3Q19 or D3Q27 lattice structures [51]. Transforming the proposed AMR routine from 2D to 3D involves the establishment of the neighbor-neighbor relations and implementation of consistent interpolation schemes. In $2 \mathrm{D}$ each block has 8 neighbors (4 in the orthogonal directions and 4 in the diagonal directions) while in 3D there are 26 neighbors for each block. Implementing an accurate and efficient interpolation scheme for different configurations of the neighboring blocks (face-to-face, edge-to-edge, or corner-to-corner) is the subject left for future study.

\section{Acknowledgment}

AF and TL acknowledge the funding received from the Department of Energy under grant number DOE 76394-003. AF would like to express his gratitude to Prof. Li-Shi Luo for enjoyable discussions and helpful advice.

\section{References}

[1] D. M. Anderson, G. B. McFadden, A. A. Wheeler, Diffuse-interface methods in fluid mechanics, Ann. Rev. Fluid Mech. 30 (1998) 139. 
[2] R. Scardovelli, S. Zaleski, Direct numerical simulation of free-surface and interfacial flow, Ann. Rev. Fluid Mech. 31 (1999) 567.

[3] D. Jamet, O. Lebaigue, N. Coutris, J. Delhaye, The second gradient method for the direct numerical simulation of liquid-vapor flows with phase change, J. Comput. Phys. 169 (2001) 624.

[4] X. He, L.-S. Luo, Theory of the lattice Boltzmann method: From the Boltzmann equation to the lattice Boltzmann equation, Phys. Rev. E 56 (1997) 6811.

[5] X. He, L.-S. Luo, lattice Boltzmann model for the incompressible NavierStokes equation, J. Stat. Phys. 88 (1997) 927.

[6] S. Chen, G. Doolen, Lattice Boltzmann method for fluid flows, Annu. Rev. Fluid Mech. 30 (1998) 329.

[7] L.-S. Luo, Unified theory of lattice Boltzmann models for nonideal gases, Phys. Rev. Lett. 81 (1998) 1618.

[8] X. He, X. Shan, G. D. Doolen, A discrete Boltzmann equation model for non-ideal gases, Phys. Rev. E 57 (1998) R13.

[9] L.-S. Luo, Theory of the lattice Boltzmann method: Lattice Boltzmann models for nonideal gases, Phys. Rev. E 62 (2000) 4982.

[10] L.-S. Luo, S. S. Girimaji, Theory of the lattice Boltzmann method: Two-fluid model for binary mixtures, Phys. Rev. E 67 (2003) 036302. 
[11] D. Yu, R. Mei, L.-S. Luo, W. Shyy, Viscous flow computations with the method of lattice Boltzmann equation, Prog. Aerosp. Sci. 39 (2003) 329.

[12] A. K. Gunstensen, D. H. Rothman, S. Zaleski, G. Zanetti, Lattice Boltzmann model of immiscible fluids, Phys. Rev. A 43 (1991) 4320.

[13] X. Shan, H. Chen, Lattice Boltzmann model for simulating flows with multiple phases and components, Phys. Rev. E 47 (1993) 1815.

[14] X. Shan, G. D. Doolen, Multicomponent lattice-Boltzmann model with interparticle interaction, J. Stat. Phys. 81 (1995) 379.

[15] M. R. Swift, E. Orlandini, W. R. Osborn, J. M. Yeomans, Lattice Boltzmann simulations of liquid-gas and binary fluid systems, Phys. Rev. E 54 (1996) 5041.

[16] X. He, S. Chen, R. Zhang, A lattice Boltzmann scheme for incompressible multiphase flow and its application in simulation of Rayleigh-Taylor instability, J. Comput. Phys. 152 (1999) 642.

[17] T. Inamuro, T. Ogata, S. Tajima, N. Konishi, A lattice Boltzmann method for incompressible two-phase flows with large density differences, J. Comput. Phys. 198 (2004) 628.

[18] T. Lee, C.-L. Lin, A stable discretization of the lattice Boltzmann equation for simulation of incompressible two-phase flows at high density ratio, J. Comput. Phys. 206 (2005) 16. 
[19] P. Lallemand, L.-S. Luo, Y. Peng, A lattice Boltzmann front-tracking method for interface dynamics with surface tension in two dimensions, J. Comput. Phys. 226 (2007) 1367-1384.

[20] A. Fakhari, M. H. Rahimian, Phase-field modeling by the method of lattice Boltzmann equations, Phys. Rev. E 81 (2010) 036707.

[21] T. Lee, L. Liu, Lattice Boltzmann simulations of micron-scale drop impact on dry surfaces, J. Comput. Phys. 229 (2010) 8045.

[22] A. Fakhari, T. Lee, Multiple-relaxation-time lattice Boltzmann method for immiscible fluids at high Reynolds numbers, Phys. Rev. E 87 (2013) 023304 .

[23] Z. Guo, C. Zheng, B. Shi, Force imbalance in lattice Boltzmann equation for two-phase flows, Phys. Rev. E 83 (2011) 036707.

[24] P. L. Bhatnagar, E. P. Gross, M. Krook, A model for collision processes in gases. I. small amplitude processes in charged and neutral onecomponent systems, Phys. Rev. 94 (1954) 511.

[25] M. J. Berger, J. Oliger, Adaptive mesh refinement for hyperbolic partial differential equations, J. Comput. Phys. 53 (1984) 484.

[26] M. J. Berger, P. Colella, Local adaptive mesh refinement for shock hydrodynamics, J. Comput. Phys. 82 (1989) 64.

[27] D. de Zeeuw, K. G. Powell, An adaptively refined Cartesian mesh solver for the Euler equations, J. Comput. Phys. 104 (1993) 56. 
[28] A. M. Khokhlov, Fully threaded tree algorithms for adaptive refinement fluid dynamics simulations, J. Comput. Phys. 143 (1998) 519.

[29] H. Ji, F.-S. Lien, E. Yee, A new adaptive mesh refinement data structure with an application to detonation, J. Comput. Phys. 229 (2010) 8981.

[30] A. Fakhari, T. Lee, Finite-difference lattice Boltzmann method with a block-structured adaptive-mesh-refinement technique, Phys. Rev. E 89 (2014) 033310.

[31] P. MacNeice, K. Olson, C. Mobarry, R. de Fainchtein, C. Packer, PARAMESH: A parallel adaptive mesh refinement community toolkit, Comput. Phys. Commun. 126 (2000) 330.

[32] R. Teyssier, Cosmological hydrodynamics with adaptive mesh refinement: A new high resolution code called RAMSES, Astron. Astrophys. 385 (2002) 337.

[33] S. Popinet, Gerris: a tree-based adaptive solver for the incompressible Euler equations in complex geometries, J. Comput. Phys. 190 (2003) 572.

[34] T. Matsumoto, Self-gravitational magnetohydrodynamics with adaptive mesh refinement for protostellar collapse, Publ. Astron. Soc. Jpn. 59 (2007) 905.

[35] U. Ziegler, The NIRVANA code: Parallel computational MHD with adaptive mesh refinement, Comput. Phys. Commun. 179 (2008) 227. 
[36] R.-L. Jiang, C. Fang, P.-F. Chen, A new MHD code with adaptive mesh refinement and parallelization for astrophysics, Comput. Phys. Commun. 183 (2012) 1617.

[37] J. Rantakokko, M. Thuné, Parallel Computing, Springer, London, pp. 147-173.

[38] J. Tölke, S. Freudiger, M. Krafczyk, An adaptive scheme using hierarchical grids for lattice Boltzmann multi-phase flow simulations, Comput. Fluids 35 (2006) 820.

[39] Z. Yu, L.-S. Fan, An interaction potential based lattice Boltzmann method with adaptive mesh refinement (AMR) for two-phase flow simulation, J. Comput. Phys. 228 (2009) 6456.

[40] A. Fakhari, T. Lee, Numerics of the lattice Boltzmann method on nonuniform grids: Standard LBM and finite-difference LBM, Comput. Fluids 107 (2015) 205-213.

[41] H. W. Zheng, C. Shu, Y. T. Chew, Lattice Boltzmann interface capturing method for incompressible flows, Phys. Rev. E 72 (2005) 056705.

[42] Y. Q. Zu, S. He, Phase-field-based lattice Boltzmann model for incompressible binary fluid systems with density and viscosity contrasts, Phys. Rev. E 87 (2013) 043301.

[43] H. Liang, B. C. Shi, Z. L. Guo, Z. H. Chai, Phase-field-based multiplerelaxation-time lattice Boltzmann model for incompressible multiphase flows, Phys. Rev. E 89 (2014) 053320. 
[44] J. W. Cahn, J. E. Hilliard, Free energy of a nonuniform system. I. Interfacial free energy, J. Chem. Phys. 28 (1958) 258.

[45] J. S. Rowlinson, B. Widom, Molecular Theory of Capillarity, Clarendon Press, Oxford, 1989.

[46] L. Zheng, T. Lee, Z. Guo, D. Rumschitzki, Shrinkage of bubbles/drops in the lattice Boltzmann equation method for nonideal gases, Phys. Rev. E 89 (2014) 033302.

[47] Y. Sun, C. Beckermann, Sharp interface tracking using the phase-field equation, J. Comput. Phys. 220 (2007) 626-653.

[48] P.-H. Chiu, Y.-T. Lin, A conservative phase field method for solving incompressible two-phase flows, J. Comput. Phys. 230 (2011) 185-204.

[49] M. Geier, A. Fakhari, T. Lee, Conservative phase-field lattice Boltzmann model for interface tracking equation, Phys. Rev. E 91 (2015) 063309.

[50] P. Lallemand, L.-S. Luo, Theory of the lattice Boltzmann method: Dispersion, dissipation, isotropy, Galilean invariance, and stability, Phys. Rev. E 61 (2000) 6546.

[51] D. d'Humiéres, I. Ginzburg, M. Krafczyk, P. Lallemand, L.-S. Luo, Multiple-relaxation-time lattice Boltzmann models in three dimensions, Phil. Trans. R. Soc. Lond. A 360 (2002) 437.

[52] J. Kim, A continuous surface tension force formulation for diffuseinterface models, J. Comput. Phys. 204 (2005) 784. 
[53] D. Lagrava, O. Malaspinas, J. Latt, B. Chopard, Advances in multidomain lattice Boltzmann grid refinement, J. Comput. Phys. 231 (2012) 4808 .

[54] A. Fakhari, Phase-Field Modeling of Multiphase Flows Using the Lattice Boltzmann Method with Adaptive Mesh Refinement, Ph.D. thesis, The City College of the City University of New York, 2015.

[55] V. V. Khatavkar, P. D. Anderson, H. E. H. Meijer, On scaling of diffuseinterface models, Chem. Eng. Sci. 61 (2006) 2364.

[56] F. Magaletti, F. Picano, M. Chinappi, L. Marino, C. M. Casciola, The sharp-interface limit of the Cahn-Hilliard/Navier-Stokes model for binary fluids, J. Fluid Mech. 714 (2013) 95-126.

[57] R. Clift, J. R. Grace, M. E. Weber, Bubbles, Drops, and Particles, Academic Press, New York, 1978.

[58] D. Bhaga, M. E. Weber, Bubbles in viscous liquids: shapes, wakes and velocities, J. Fluid Mech. 105 (1981) 61-85.

[59] A. Fakhari, M. H. Rahimian, Simulation of an axisymmetric rising bubble by a multiple relaxation time lattice Boltzmann method, Int. J. Mod. Phys. B 23 (2009) 4907-4932.

[60] J. Han, G. Tryggvason, Secondary breakup of axisymmetric liquid drops. I. Acceleration by a constant body force, Phys. Fluids 11 (1999) 3650.

[61] A. Fakhari, M. H. Rahimian, Simulation of falling droplet by the lattice 
Boltzmann method, Commun. Nonlinear Sci. Numer. Simulat. 14 (2009) $3046-3055$.

[62] A. Fakhari, M. H. Rahimian, Investigation of deformation and breakup of a falling droplet using a multiple-relaxation-time lattice Boltzmann method, Comput. Fluids 40 (2011) 156-171.

[63] M. Jalaal, K. Mehravaran, Fragmentation of falling liquid droplets in bag breakup mode, Int. J. Multiphase Flow 47 (2012) 115-132.

[64] X. Zheng, J. Lowengrub, A. Anderson, V. Cristini, Adaptive unstructured volume remeshing - II: Application to two- and three-dimensional level-set simulations, J. Comput. Phys. 208 (2005) 626-650.

[65] F. Blanchette, T. P. Bigioni, Partial coalescence of drops at liquid interfaces, Nat. Phys. 2 (2006) 254.

[66] P. Yue, C. Zhou, J. J. Feng, A computational study of the coalescence between a drop and an interface in Newtonian and viscoelastic fluids, Phys. Fluids 18 (2006) 102102.

[67] R. H. Davis, J. F. Schonberg, J. M. Rallison, The lubrication force between two viscous drops, Phys. Fluids A 1 (1988) 77.

[68] G. Bozzano, M. Dente, Modelling the drop coalescence at the interface of two liquids, Comput. Chem. Eng. 35 (2011) 901-906.

[69] W. Tauber, S. O. Unverdi, G. Tryggvason, The nonlinear behavior of a sheared immiscible fluid interface, Phys. Fluids 14 (2002) 2871. 
[70] H. D. Ceniceros, A. M. Roma, Study of the long-time dynamics of a viscous vortex sheet with a fully adaptive nonstiff method, Phys. Fluids 16 (2004) 4285.

[71] X. Yang, X. Zhang, Z. Li, G.-W. He, A smoothing technique for discrete delta functions with application to immersed boundary method in moving boundary simulations, J. Comput. Phys. 228 (2009) 7821.

[72] H. Yu, L.-S. Luo, S. S. Girimaji, LES of turbulent square jet flow using an MRT lattice Boltzmann model, Comput. Fluids 35 (2006) 957-965.

[73] P. R. Redapangu, K. C. Sahu, S. P. Vanka, A study of pressure-driven displacement flow of two immiscible liquids using a multiphase lattice Boltzmann approach, Phys. Fluids 24 (2012) 102110.

[74] L.-P. Wang, O. Ayala, H. Gao, C. Andersen, K. L. Mathews, Study of forced turbulence and its modulation by finite-size solid particles using the lattice Boltzmann approach, Comput. Math. Appl. 67 (2014) 363380 . 